US Army Corps of Engineers ${ }_{\circledast}$ Engineer Research and Development Center

\title{
Fusion of Spectral Data from Multiple Handheld Analyzers (LIBS, XRF and Raman) for Chemical Analysis and Classification of Soil
}

Russell S. Harmon, Richard R. Hark, Chandra S. Throckmorton,

John R. Plumer, Jan M.H. Hendrickx, J. Bruce Harrison, and

Karen A. Harmon 
The U.S. Army Engineer Research and Development Center (ERDC) solves the nation's toughest engineering and environmental challenges. ERDC develops innovative solutions in civil and military engineering, geospatial sciences, water resources, and environmental sciences for the Army, the Department of Defense, civilian agencies, and our nation's public good. Find out more at www.erdc.usace.army.mil.

To search for other technical reports published by ERDC, visit the ERDC online library at https://erdclibrary.on.worldcat.org/discovery. 


\section{Fusion of Spectral Data from Multiple Handheld Analyzers (LIBS, XRF and Raman) for Chemical Analysis and Classification of Soil}

Russell S. Harmon, Richard R. Hark, John R. Plumer, and Karen A. Harmon

JRPlumer Associates, LLC;

Gilford, NH 03249

Chandra S. Throckmorton

Signal Analysis Solutions, LLC

22 Piney Grove Road

Bahama, NC 27503

Jan M.H. Hendrickx and J. Bruce Harrison

Soil Hydrology Associates, LLC

1113 Valley View Drive

Los Lunas, NM 87031

Final report

Approved for public release; distribution is unlimited.

Prepared for U.S. Army Engineer Research and Development Center Vicksburg, MS 39180

Under ERDC T-15 Program, Project 474428, "Support of US Army Global Military Objectives: LIBS for Military Surveys"

Monitored by Cold Regions Research and Engineering Laboratory U.S. Army Engineer Research and Development Center Hanover, NH 03755 


\section{Abstract}

An 18-month multidisciplinary project was undertaken by JRPlumer \& Associates, LLC and four subcontractors that had three technical objectives: (i) to upgrade current handheld technology for chemical analysis by X-ray fluorescence spectroscopy (XRFS), Raman spectroscopy (RS), and laser-induced breakdown spectroscopy (LIBS); (ii) to design a multisensor system based on these technologies for the rapid, in-situ chemical analysis of soils and other materials of military interest; and (iii) to investigate the classification/discrimination performance benefit that might be achieved through advanced signal pre-processing and data fusion with XRFS, RS, and LIBS analyses acquired for four suites of natural soils. Accomplishments of the program in the latter area are described in this report.

DISCLAIMER: The contents of this report are not to be used for advertising, publication, or promotional purposes. Citation of trade names does not constitute an official endorsement or approval of the use of such commercial products. All product names and trademarks cited are the property of their respective owners. The findings of this report are not to be construed as an official Department of the Army position unless so designated by other authorized documents. 


\section{Contents}

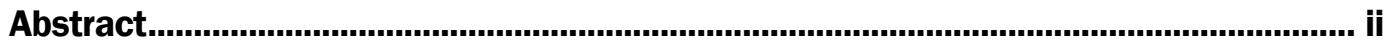

Preface

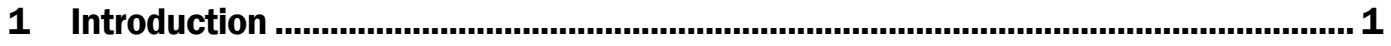

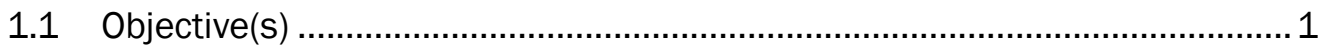

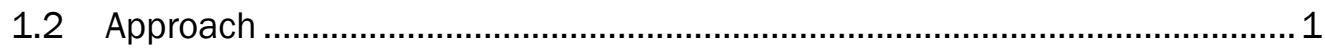

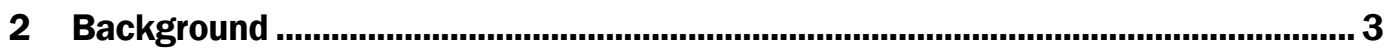

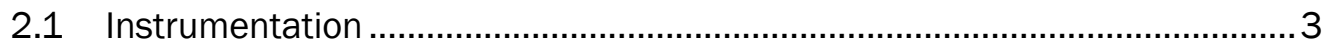

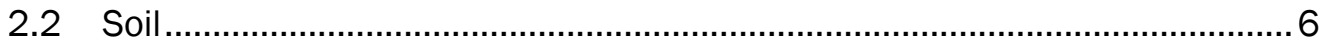

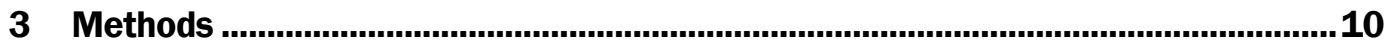

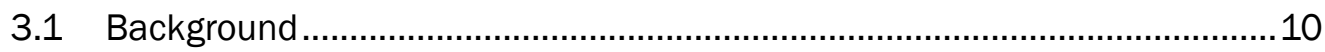

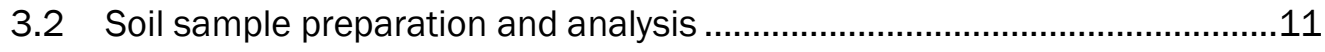

4 Signal Processing, Statistical Analysis, and Data Fusion......................................14

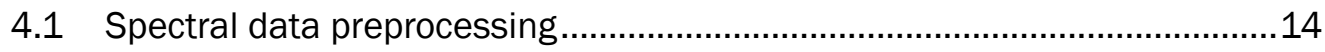

4.1.1 Spectral truncation (XRFS) and normalization (XRFS, LIBS, RS) ........................ 14

4.1.2 Argon spectral line removal (LIBS)................................................................ 14

4.1.3 Baseline correction (LIBS) ....................................................................... 15

4.1.4 Spectra culling (LIBS) ........................................................................... 16

4.1.5 Signal-to-Noise Ratio (SNR) .................................................................... 16

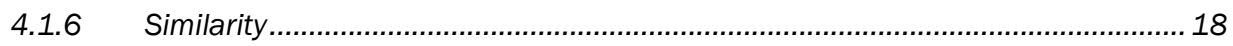

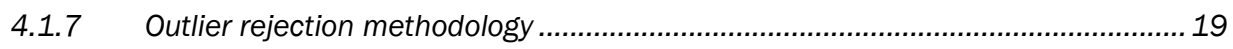

4.1.8 Outlier rejection results .................................................................................21

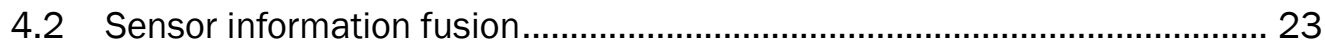

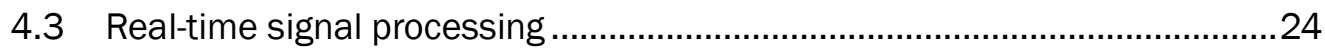

5 Data Analysis and Spectral Fusion Results and Discussion ..................................27

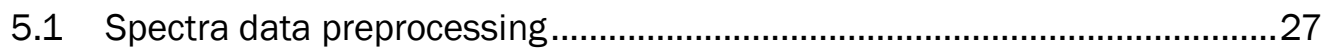

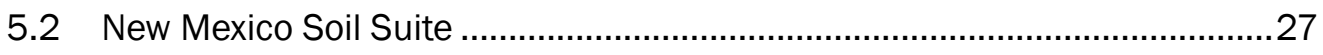

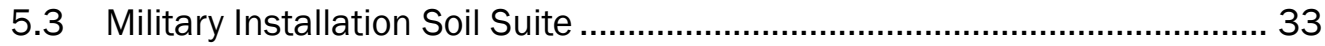

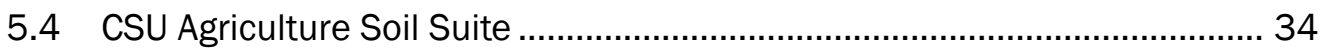

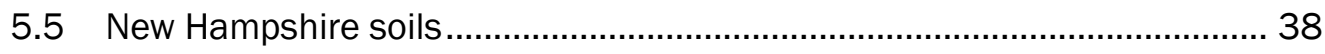

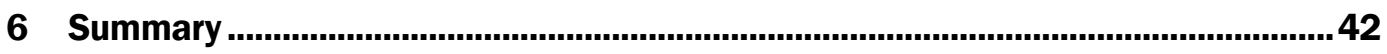

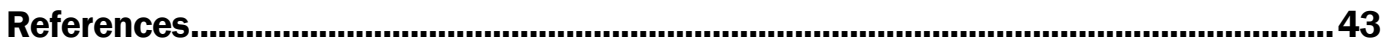

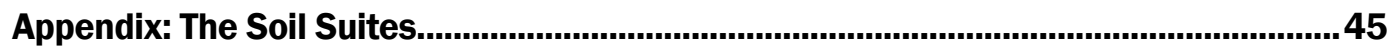

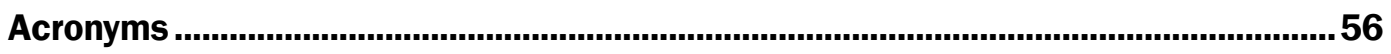

Report Documentation Page 


\section{Figures and Tables}

\section{Figures}

Figure 1. The SciAps commercial-off-the-shelf handheld analyzers for X-ray fluorescence spectroscopy (left), Raman spectroscopy (center), and laserinduced breakdown spectroscopy (right), with the performance attributes of each analyzer noted.

Figure 2. Example XRFS spectrum for the playa soil from White Sands, NM, showing the presence of $\mathrm{Ca}, \mathrm{Sr}, \mathrm{S}$ and $\mathrm{Fe}$.

Figure 3. Raman spectrum for the playa soil from White San.

Figure 4. LIBS broadband spectrum for the playa soil from White Sands, NM, showing presence of $\mathrm{Ca}$ and $\mathrm{Sr}$ together with the minor presence of $\mathrm{Si}$ and $\mathrm{Na}$.

Figure 5. Spectral variability plot degree of homogeneity/heterogeneity between the different locations analyzed on soil pellets for 14 soil standards.

Figure 6. Difference between typical classification (left) and outlier rejection (right).

Figure 7. Implementation of the NOTA analytical process.

Figure 8. XRFS outlier rejection rates for different data sets when the nonNOTA data set is the military installation soil suite.

Figure 9. Rejection rates at the optimal operating point for which the costs of the two types of errors (failing to reject NOTA samples and rejecting correctly

classified non-NOTA samples) were assumed equal.

Figure 10. Flow diagram for XRFS-RS-LIBS spectral fusion procedure.

Figure 11. Architecture diagram demonstrating expected software operation. The software consists of three main modules that can be exchanged as needed (e.g. advancement in data acquisition). In addition, the data processing module will likely contain modules that can be exchanged to change operation (e.g. classifier, preprocessing).

Figure 12. Classification accuracy for the New Mexico soil suite based on consideration of spectra for XRFS, RS, and LIBS; fusion of spectra for RS + XRFS, LIBS + XRFS; and for RS + LIBS + XRFS for spectral data collected in Jan 2019, Mar/Apr 2019, and for data fusion from both dates.

Figure 13. Single analyzer classification results for three different methods of training.

Figure 14. Classification results for XRFS and LIBS fusion for three different methods of training. For each data collection, XRFS data is paired with that from either the JRPA LIBS analyzer or the SciAps LIBS analyzer.

Figure 15. Classification results for fusing the Raman, XRFS, and LIBS sensors using three different methods of training. For each data collection, Raman and XRF are paired with either the JRPA LIBS sensor or the SciAps LIBS sensor.

Figure 16. Classification accuracy for the military installation soils based on RS, XRFS, and LIBS data; fused 2-analyzer data, and fused 3-analyzer data. 
Figure 17. Classification accuracy for the CSU classification schema based on consideration of spectra for Raman spectroscopy - RS (1), X-ray fluorescence spectroscopy - XRFS (2), laser-induced breakdown spectroscopy - LIBS (3), fused spectra for RS + XRFS (4), RS + LIBS (5), and XRFS + LIBS (6), RS + XRFS + LIBS spectra (7), fused spectra + XRF quantitative analyses (8), and fused spectra + XRF quantitative analyses + laboratory chemical analyses (9).

Figure 18. Independent feature accuracy per CSU soil classification schema. Features are sorted on a per schema basis, greatest accuracy to least accuracy. The legends indicate classification performance that could be achieved by always selecting the class with the most samples.

Figure 19. Principal component analysis score plots for XRFS (upper left), LIBS (lower left), RS (upper right), and chemistry (lower right) for the soil series of CSU soil suite represented by multiple samples

Figure 20. Principal component analysis scores plot from laboratory analysis of the Lebanon/CRREL and Belmont soil sets.

Figure 21. Principal components scores plot from laboratory analysis of the Belmont soil pits

Figure 22. Classification accuracy for the New Hampshire soil sets from Belmont/Bean Hill (left) and Lebanon/CRELL (right)

Figure 23. Principal component analysis scores plot for fused XRFS and LIBS spectral data sets for in-situ analysis of the three Belmont/Bean Hill soil pits.

\section{Tables}

Table 1. Comparative capabilities of SciAps handheld XRFS, RS, and LIBS analyzers.

Table 2. Perceived advantages of an integrated multi-analyzer system. 5

Table 3. Number of samples per class for each collection date.. .29

Table 4. Classification schema for CSU soil suite characterization. 


\section{Preface}

This study was conducted for ERDC T-15 Program under 474428, "Support of US Army Global Military Objectives: LIBS for Military Surveys.” Parts of this program were funded under ERDC contract W913E518Co011 issued to JRPlumer \& Associates LLC. The technical monitor was Dr. Jay Clausen, CEERD-RN.

The work was monitored by the Biogeochemical Sciences Branch of the Research and Engineering Division, U.S. Army Engineer Research and Development Center Cold Regions Research and Engineering Laboratory (ERDC-CRREL). At the time of publication of this report Mr. Jimmy D. Horne was Division Chief of CEERD-RR and the Director of ERDCCRREL was Dr. Joseph L. Corriveau.

The Commander of ERDC was COL Teresa A. Schlosser and the Director was Dr. David W. Pittman. 


\section{Introduction}

An 18-month multidisciplinary effort was undertaken by JRPlumer \& Associates, LLC with three technical objectives: (i) to upgrade current commercial handheld technology for chemical analysis by X-ray fluorescence spectroscopy (XRFS), Raman spectroscopy (RS), and laser-induced breakdown spectroscopy (LIBS); (ii) to design a multianalyzer system based on these technologies for the rapid, in-situ chemical analysis of soils and other materials of military interest; and (iii) to investigate the classification/discrimination performance benefit that might be achieved through signal pre-processing and data fusion using XRFS, RS, and LIBS analyses of natural soils. This initiative was supported by subcontracts from JRPlumer \& Associates, LLC to SciAps, Inc., Signal Analysis Solutions, LLC, SoilHydrology Associates, LLC, and Applied Spectra, Inc. Accomplishments of this multidisciplinary R\&D program pertain to three technical areas: Technology Development and Design, Soil and Military Material Analysis; and Signal Processing, Chemometric Analysis, and Data Fusion. Details of work completed under the first two program areas are commercial proprietary, so this report describes the results only from the third program area.

\subsection{Objective(s)}

A central hypothesis of the multisensor concept is that integration of three chemical analyzers with largely complementary capabilities would result in an operational performance surpassing that achievable by any of the individual handheld analyzers. Thus, the overarching program objective was to evaluate this hypothesis.

\subsection{Approach}

The program vision was that a multianalyzer capability would permit discrimination of materials of interest presently beyond the capability of contemporary field-portable analyzers and at a price point significantly lower than laboratory instrumentation. Thus, spectral data preprocessing and fusion of the processed data streams from the individual analyzers were studied to assess the extent to which classification/discrimination performance could be improved. Anticipated performance enhancement would 
be a consequence of utilizing an integrated approach to data preprocessing, fusion of processed data streams from the three analyzer types to be joined in the multi-analyzer system, and state-of-the-art chemometric analysis employing advanced statistical analysis, pattern recognition, and machine learning techniques for material classification/discrimination. As another step in laying the foundation for a multianalyzer system, algorithms were developed for processing the analyzer data streams and undertaking the chemometric analysis in real time.

A soil analysis component of the program was needed to baseline the performance of the three individual commercial analyzers and to provide the data needed for the signal processing and data analytics research. Four suites of natural soils were acquired and then analyzed using the commercial XRFS, RS, and LIBS analyzers individually. Various approaches to the pre-processing of the LIBS data, which is characterized by high shot-toshot spectral intensity variation, were examined. Then, the processed data for each sample analyzed was fused with the XRFS and RS data streams for that samples, with the results for the fused results for the integrated analyzer data streams compared with that from the individual analyzers. 


\section{Background}

\subsection{Instrumentation}

Until quite recently, chemical analysis for material characterization has been possible only in the laboratory. Consequently, laboratory analysis of materials collected in the field, typically tends to be limited to a small number of samples that are analyzed using specialized instrumentation such as inductively coupled plasma mass spectrometry, high performance liquid chromatography, or gas chromatography. These processes are labor intensive, time-consuming, and typically very costly because individual samples must be collected and packaged on-site in the field, transported to the laboratory, and then processed for analysis on what can be very expensive instrumentation. However, with the recent advent of portable single sensor instrumentation for chemical analysis in the field, a technological opportunity existed to create a novel and unique field characterization and forensic capability for the rapid, in-situ analysis of both soil and a wide range of materials of military interest undisturbed in the field under ambient environmental conditions.

Thus, a program of coordinated interdisciplinary research, development, testing, and evaluation (RDTE) was developed to design, integrate, and construct a compact, and lightweight multianalyzer system for deployment by an individual or on a robotic unmanned ground or aerial vehicle. A 3part program was structured that would first enhance and refine the current handheld commercially available sensors for XRFS, RS, and LIBS, design an integrated multianalyzer system, develop and test a data fusion process, and finally fabricate a multi-analyzer system that would be demonstrated and validated through both laboratory and field testing. The first three parts of this program were funded under ERDC contract W913E518Coo11 issued to JRPlumer \& Associates LLC on 20 September 2018 for an 18 months period of performance.

$\mathrm{X}$-ray fluorescence spectroscopy (XRFS) is a technique for determination of bulk chemical composition that uses X-ray-induced fluorescence to compositionally interrogate a broad ( $\sim \mathrm{mm}$ size) area of a material surface and is particularly efficacious for transition metals and other heavy elements. Raman spectroscopy (RS) observes the inelastic scattering of monochromatic laser light from a material surface to measure the frequency of molecular vibrational and rotational modes to acquire a structural fingerprint 
by which different substances can be identified. RS analyzers can be configured to have either a point or broad area interrogation capability. Laserinduced breakdown spectroscopy (LIBS) utilizes a pulsed laser to ablate a very small (10s $\mu \mathrm{m}$ size) area on the surface of a material and determine its chemical composition through spectral analysis of emitted light. It is particularly sensitive to light elements and is also capable of continuous depth profiling into a sample. Thus, it can also be used to 'clean' a sub$\mathrm{mm}$-size area on a sample surface prior to analysis so that underlying layers can be compositionally interrogated once any surface coating, crust, or corrosion is removed.

Figure 1. The SciAps commercial-off-the-shelf handheld analyzers for X-ray fluorescence spectroscopy (left), Raman spectroscopy (center), and laser-induced breakdown spectroscopy (right), with the performance attributes of each analyzer noted.

\section{The Handheld Analyzers}

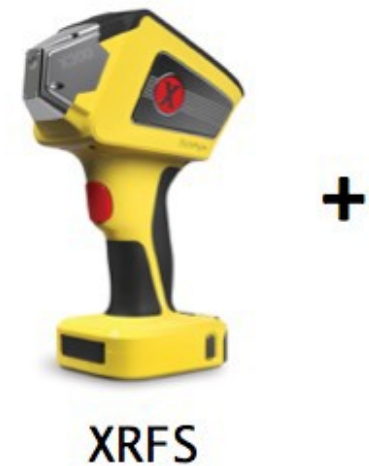

Detection and quantitative analysis of most elements from $\mathrm{Mg}$ to $\mathrm{U}$

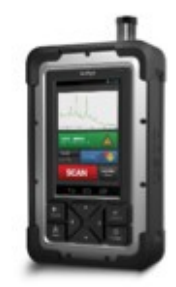

RS

Molecular detection for mineral and organic material identification

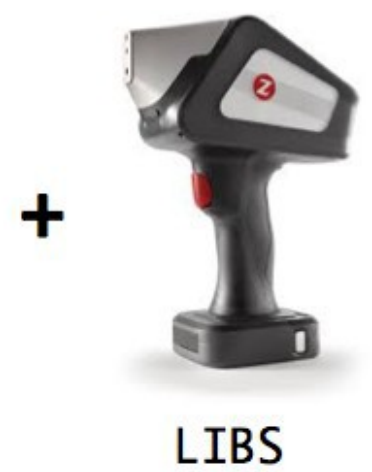

Analysis of material in any form solid, liquid, or gas; Excellent for the detection of light elements from $\mathrm{H}$ through $\mathrm{Na}$ and elements of low ionization potential

Each of these three technologies for chemical analysis - XRFS, RS, and LIBS, are presently commercially available as handheld analyzers for use outside the laboratory (Figure 1) and have common performance attributes like minimally-destructive analysis and rapid data collection and processing. It is well recognized that these different analytical technologies have different elemental sensitivities and analytical accuracies. However, each modality has its own unique functional competency (Table 1), such as differential sensitivity to a wide range of elements, point versus area analysis, surface analysis versus depth profiling and analysis of elemental versus 
molecular compositions. It has also been observed that by synthesizing raw data from several sources and processing the integrated data using the tools of multivariate statistical analysis (i.e. data fusion) it is possible to generate more meaningful information than can be generated by using data from any single source and that such fused information that can be of enhanced value. For example, data fusion for different kinds of analytical instruments can reduce limits of detection and improve uncertainty, whereas the fusion of information from different types of sensors with different physical characteristics can enhance insight about the environment being observed.

Table 1. Comparative capabilities of SciAps handheld XRFS, RS, and LIBS analyzers.

\begin{tabular}{|l|c|c|c||}
\hline \multicolumn{1}{|c|}{ CAPABILITY } & XRF & RS & LIBS \\
\hline & & & solid, liquid, \& gas \\
\hline material & solid & solid, liquid, \& gas & minimally-destructive \\
\hline sample impact & non-destructive & non-destructie & minimal \\
\hline sample preparation & minimal & minimal & enhanced in buffer gas \\
\hline measurement environment & in air & in air & few mm \\
\hline analytical spot size & $\sim 5-10 \mathrm{~mm}$ & few minutes & seconds \\
\hline measurement time & few minutes & no & yes \\
\hline depth analysis & no & no & yes \\
\hline spatial mapping & no & molecular detection & light elements H-Na \\
\hline strengths & elemental coverage Mg-U & mineral identification & $\begin{array}{c}\text { quantification requires matrix- } \\
\text { matched standards }\end{array}$ \\
\hline
\end{tabular}

Table 2. Perceived advantages of an integrated multi-analyzer system.

- Rapid on-site detection and discrimination of materials of interest by trained field personnel (not highly trained technicians)

\begin{tabular}{|ll|}
\hline - & Undertake qualitative detection or quantitative analysis \\
\hline - & Low cost (some 10x less than laboratory equivalents) \\
\hline - & Small size, weight, power requirements to enable use on remotely-operated \\
\hline - & Deployment for interrogation of inaccessible and denied area \\
\hline
\end{tabular}

The integration of the three analyzers into a single multi-analyzer system would greatly expand current technological capability for real-time chemical analysis in the field under ambient environmental conditions (Table 2). 
There is strong potential for technology transition spinoff for other applications. For example, one such possibility would be its use for field forensics by both the civil and military law enforcement communities.

\subsection{Soil}

To fully understand and exploit the analytical benefit a field-portable multianalyzer capability, it is important to understand the chemistry of target of interest for this program - natural soil.

Soil is defined by the United State Department of Agriculture (USDA) National Resources Conservation Service (NRCS) as natural unconsolidated material on the immediate surface of the Earth comprised of solids (minerals and organic matter), liquid, and gases that serves as the growth medium of land plants (NRCS 1999). Soils and their internal horizons differ from one another and depend on five soil-forming factors - geologic parent material, climate, topography, biological factors associated with the flora and fauna, and time (Jenny 1994). There is a wide variety of geological units, climates, floras and faunas, and topography across the United States, while the factor time spans many orders of magnitude from a few to hundreds of thousands of years. For example, in New Mexico the gypsiferous sand soil at White Sands National Monument can be as young as a few weeks, whilst the ancient gravelly sandy loam soil on Sedillo Hill has an age of about 750,000 years.

Eight chemical elements (O, Si, $\mathrm{Al} \mathrm{Fe}, \mathrm{Mg}, \mathrm{Ca}, \mathrm{Na}, \& \mathrm{~K})$ comprise the bulk of the inorganic component of soils. Ions of these elements combine in various ratios to form different minerals. Another $80+$ elements naturally occur in soil in minor and trace quantities and still others can be present as anthropogenic contaminants. Soils are chemically different from the rocks and minerals from which they form in that soils contain less of the water-soluble weathering products, $\mathrm{Ca}, \mathrm{Mg}, \mathrm{Na}$, and $\mathrm{K}$ and more of the relatively insoluble elements such as Fe and Al. Old, highly weathered soils normally have high concentrations of aluminum and iron oxides. The organic fraction of the near-surface soil, which is composed predominantly of, carbon, hydrogen, oxygen, nitrogen and smaller quantities of sulfur and other elements, usually comprises $<6 \%$ of the soil mass by weight and much less at depth. Yet, despite its small quantity it has a great influence on soil chemical properties. The organic fraction enhances soil aggregation 
and structure, enhances soil water retention, and increases cation exchange capacities, while also serving as a reservoir for the plant essential nutrient elements (e.g. N, P, and S).

Soils are classified based on physical and chemical properties in their different horizons (e.g. composition, color, texture, structure, etc.) from the surface to two meters depth. Currently, the NRCS soil database for the United States

(https://www.nrcs.usda.gov/wps/portal/nrcs/detail/soils/survey/class/da ta/?cid=nrcs142p2_053586) contains more than 20,000 detailed soil series descriptions that reflect the wide range of the five soil-forming factors across the country. The NRCS soil taxonomy establishes hierarchies of classes to permit the relationship among soils and between soils, and the factors responsible for their character, to be understood. The soil series is the lowest category within soil taxonomy. All soils within a single series have uniform differentiating characteristics and arrangement of horizons. This does not mean that all soils within a series are identical; it does mean that they have a similar sequence of horizons, but the horizons may be of different thickness, color, structure, within prescribed limits. All the soils within a series will have developed in the same kind of parent material with comparable drainage characteristics and will be of similar age. The effects of climate and biological activity will have been very similar. Consequently, the soils within a series exhibit like properties and respond in like fashion to usage or manipulation.

To understand the soil landscape and how it is portrayed on soil maps it is important to understand what is being mapped (soil taxonomic units) and how their distribution is recorded on a soil map (soil mapping units). All soils in the U.S. are classified according to an established soil taxonomy that has six categories from highest to lowest: Order, Suborder, Great Group, Subgroup, Family, and Series. The classification becomes more exclusive from Soil Orders to Soil Series. There are 12 Soil Orders and over 25,000 Soil Series, generally the spatial distribution of Soil Series is what is portrayed on NRCS soil maps.

The single most important factor determining the accuracy of any soil map is the map scale. Most intensive agricultural areas have soil maps at a scale of 1:20,000 or less. All other areas, also known as rangeland areas, have soil maps at a scale of 1:50,000 or greater. The smallest unit that can be defined on a map at this scale is approximately 20 acres. 
It is important to note that the method for mapping soils in rangelands areas of the western US differs from that in more intensively farmed regions. In agricultural areas, soil map unit boundaries are identified primarily by the change in soil properties determined from soil auguring and, more recently, remote sensing tools such as electromagnetic induction meters. By contrast, rangeland soil maps are based largely on the interpretation of aerial photographs to identify proxy data that has been determined reflect changes in soil properties, such as changes in landform, parent materials, vegetation, etc. The initial identification of soil map units is validated with a very small number of soil observations, commonly $4-5 / \mathrm{km}^{2}$. At the rangeland scale, many soil map units are described as either complex or compound Soil Mapping Units (SMU), compound SMU's predominantly consist of two Soil Series whereas complex SMU's can contain more than two Soil Series. Furthermore, in the description of the map units there is no indication how the two or more Soil Series are distributed within the unit.

Unit boundaries on all soil maps are shown as fixed (or definite) boundaries that indicate the immediate change from one Soil Series to the adjoining Soil Series. However, two very different types of soil map unit boundaries can be observed in the field - well-defined boundaries and gradational boundaries. Well-defined boundaries are those where soil properties abruptly change across a landform or due to a change in lithology. Such boundaries are easily identified by landform or lithological relationships and the boundary does not change over time. By contrast, gradational boundaries can change over time and may change according to the soil properties being considered. Most of the soil map unit boundaries for maps at a scale of 1:50,000 and above are boundaries based on landform and/or lithological changes.

The NRCS has identified and mapped the over 20,000 different Soil Series across the United States at different levels of classification from Family, to Subgroup, Great Group, Suborder and Order. The NRCS soil taxonomy divides natural soils into 12 Orders that exhibit only small differences in the kinds and relative strengths of processes that tend to develop their internal soil horizons: Alfisols (10\% - in semiarid to moist areas), Andisols (1\% - in cool areas with moderate to high rainfall, Aridisols (12\% too dry for mesophytic plants), Entisols (16\% - little to no pedogenic development), Gelisols ( $9 \%$ - permafrost near the soil surface), Histosols (1\% - high organic content without permafrost), Inceptisols (17\% - moderate development in 
semi-arid to humid environments), Mollisols (moderate to pronounced soil moisture deficit), Oxisols (8\% - highly weathered soils in subtropical and tropical regions), Spodosols (4\% - acid and infertile), Ultisols (8\% acid soils in humid regions), and Vertisols (2\% - high content of expanding clay minerals). The NRCS classification recognizes 64 Suborders, 300 Great Groups, and more than 2400 Subgroups.

Thus, a major challenge for this project was how to select a representative suite of the most common soils that might be encountered by the military during operations in the field. One option was to select soils that represent the 12 soil orders of the USDA soil taxonomy. A Soil Order is the category with the highest level of generalization and abstraction, and each Soil Order is very heterogeneous with respect to its physical and chemical properties that are not considered in the differentiation (NRCS 1999). As a consequence, knowing only the Order of a soil is not sufficient to allow for scientific repeatability of compositional measurements. Soil Series are the most homogeneous classes in the system of taxonomy of the NRCS. They are the lowest and most homogeneous category of the national soil classification system and provide a detailed record of soil properties needed to prepare soil evaluations for different applications in agriculture, construction, roads, etc. An official NRCS Soil Series description includes information on location, taxonomic classification, a detailed soil profile description, location of the typical soil profile, the range of descriptive characteristics, competing series, geographic setting, geographically associated soils, drainage and permeability, use and vegetation, distribution and extent, and additional data. Therefore, a soil sample from an officially recognized Soil Series taken at the "location of the typical soil profile", represents a unique soil that is readily accessible, should additional sampling be needed in the future for further testing and confirmation. However, it was not possible to select a small set of representative soils from the $25,000+$ soil series in the United States to cover the full range of soil characteristics because any stratification scheme will necessarily fall short. 


\section{Methods}

\subsection{Background}

JRPA undertook a capabilities performance investigation of the current COTS handheld analyzers for XRFS, RS, and LIBS to understand performance through a detailed program of comparative detection and quantification of chemical elements and molecular species in soils. Two types of soils were used for this purpose: (1) Standard Reference Materials (SRMs) and (2) natural soils from across North America. A listing of these soils and standards is provided in the on-line archive for this project in the JRPA Multisensor Project folder on the Open Science Framework tool (https://osf.io/z3wgk/). Although SRMs can serve as consistent calibration points for sensor performance, previous work by Hendrickx and his colleagues on the detection of improvised explosive devices (e.g., Das et al. 2001; Miller et al. 2004; Van Dam et al. 2004; Hendrickx et al. 2006) unambiguously corroborated that more will be learned from sensor development research using representative field soils than commercially available standard materials. The latter are unrepresentative of the wide range of natural soils that are encountered in the field. Thus, test results in these media will not necessarily give a realistic evaluation on the potential and limitations of a novel sensor for field soil analysis.

A total of 272 natural soils were acquired from four sources for the project analytical work. These are follows: (i) 50 rangeland soil samples from central New Mexico, (ii) 21 soil samples from two sites in west-central New Hampshire, (iii) a suite of 144 agricultural soils from across the United States and Canada provided by Colorado State University, and (iv) 58 soils from small arms ranges on military installations in Massachusetts, Virginia, Georgia, Idaho, Washington, and Alaska provided by USACE-ERDCCRREL. Each soil suite is described in the Appendix to this report. Together, these soil suites met the following criteria:

(i) The soils should be representative of a particular area;

(ii) The soils chosen should represent different climatic settings across the country (from semi-arid to humid, because climate is an important soil forming factor); 
(iii) The soils should represent different geologic parent materials, because parent material also is an important soil forming factor;

(iv) The soils should represent different ages because time is another important soil forming factor;

(v) The soil should be relatively thick and have distinguishable A and B horizons, so that well-defined, homogeneous samples can be collected; and

(vi) The soils should represent more than half of the twelve soil orders.

\subsection{Soil sample preparation and analysis}

The natural soil samples for the project were sieved with \#10 (2 mm sieve), milled with a ceramic mortar and pestle for 1 minute, and then aliquots were hydraulically pressed with 1 ton of pressure for 2 minutes into pellets for analysis. Soil standards, received as homogeneous powders, were hydraulically pressed into pellets in the same manner as the natural soils.

XRFS analyses were undertaken using a SciAps X-25o handheld analyzer. The X-250 utilizes a rhodium X-ray tube with $4 \mathrm{~mm}$ spot size and $20 \mathrm{~mm}$ silicon drift detector (SSD) with $135 \mathrm{eV}$ resolution that has a FWHM of 5.95 for the manganese K-alpha line. An energy-to-channel calibration performed at the beginning of operation and approximately every 30 minutes thereafter. Three separate sequential measurements of 40 seconds time were made with three different beam parameters $(50 \mathrm{KeV}, 60 \mathrm{~mA}$, no filter; $40 \mathrm{KeV}, 60 \mu \mathrm{A}$, no filter; and $15 \mathrm{KeV} 80 \mu \mathrm{A}, 100 \mathrm{~mm} \mathrm{Al} \mathrm{filter)} \mathrm{at} \mathrm{each}$ of three locations on each soil pellet using the SciAps internal 'soil mode' calibration based on the analysis of $40 \mathrm{SRMs}$. This provided quantitative data for 17 elements of interest (P, S, K, Ca, Ti, Fe, V, Cr, Mn, Ni, Zn, Rb, $\mathrm{Sr}, \mathrm{Zr}, \mathrm{Sn}, \mathrm{Sb}, \mathrm{Ba}$ ), with some other elements of interest (Co, Mo, $\mathrm{Cd}, \mathrm{Pb}$, $\mathrm{U}, \mathrm{Th}$ ) consistently below the limit of detection in the soil suites analyzed.

RS analyses were obtained with a SciAps Chem-200 Raman analyzer. This instrument utilizes a 785 nanometer $(\mathrm{nm})$ laser that produces 500 milliwatt ( $\mathrm{mW}$ ) (maximum power). Spectra were collected over a wavenumber range of 200-1800 cm-1 through accumulation of 15, 1.2 second accumulations at three locations on each soil pellet after calibration using a benzonitrile standard. Raw, unprocessed (i.e. non-baseline corrected) spectra were used for the data analysis. 
LIBS analyses were performed using a SciAps Z-300 LIBS instrument which contains a $1064 \mathrm{~nm}$ neodymium-doped yttrium aluminum garnet (Nd:YAG) laser that produces a 50-100 micrometer $(\mu \mathrm{m})$ diameter beam on the sample surface. The laser was operated at 5-6 millijoule (mJ), with a 1 nanosecond (ns) pulse duration, and a 10 hertz (Hz) firing rate. The three spectrometer-charged couple device pairs, covering the spectral range of spectral range of 190 to $950 \mathrm{~nm}(\mathrm{FWHM}=0.20 \mathrm{~nm}$ at $<365 \mathrm{~nm})$, $0.25 \mathrm{~nm}$ from 365 to $620 \mathrm{~nm}$, and $0.40 \mathrm{~nm}$ at $>620 \mathrm{~nm}$ ) had a gate width of 1 millisecond a gate delay of $\sim 650 \mathrm{~ns}$. These analyses were collected under Ar gas flow using three data collection laser shots after two cleaning shots over three $4 \times 2$ grids of 50-100 nm diameter at 3 locations on each soil pellet to generate 72 spectra, each 22,500 data points per spectrum, for each sample after on-board calibration every 10 minutes. The instruments were wavelength calibrated at the beginning of operation and periodically thereafter at approximately 30-minute intervals. A second Z-300 LIBS analyzer provided by SciAps was used to acquire a comparable data set for instrument performance comparison.

XRFS and LIBS spectra were preprocessed using z-scoring (mean subtraction and standard deviation division) normalization before further processing and fusion. XRFS spectra were truncated to be the same length and LIBS spectra had Ar spectral lines removed and baseline corrected. No normalization was applied to the XRFS quantitative data and RS spectra were utilized as collected.

Examples of typical XRFS, RS, and LIBS spectra obtained during this project are presented in Figures 2-4, in this case for the playa lake soil at the White Sands, NM sampling locality. These three figures illustrate clearly that each of the handheld analyzers not only records similar compositional information, as expected, but also can contribute unique information about the composition of a soil sample. 
Figure 2. Example XRFS spectrum for the playa soil from White Sands, NM, showing the presence of $\mathrm{Ca}, \mathrm{Sr}, \mathrm{S}$ and $\mathrm{Fe}$.

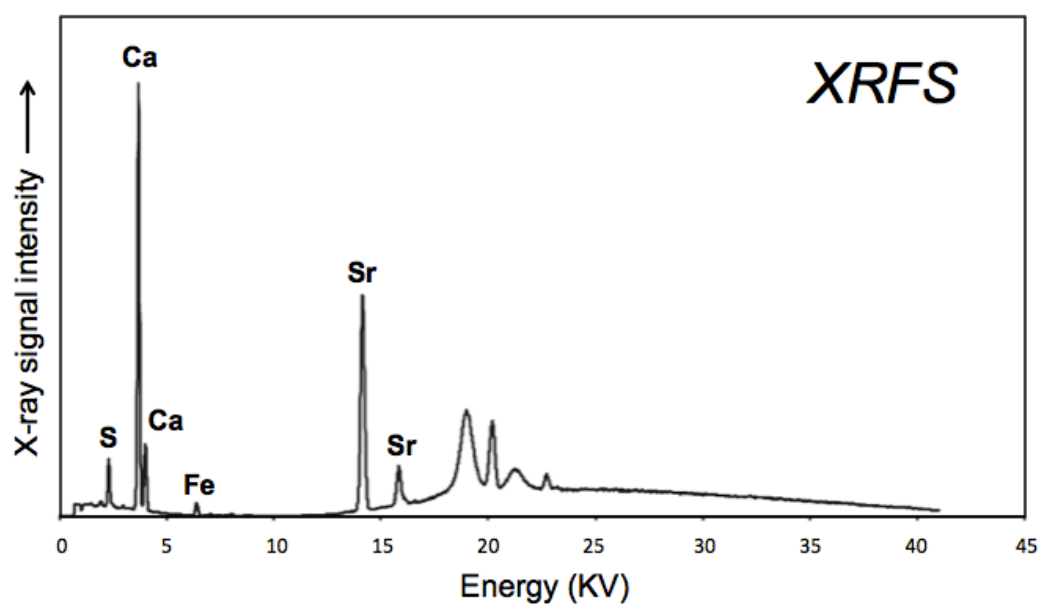

Figure 3. Raman spectrum for the playa soil from White San.

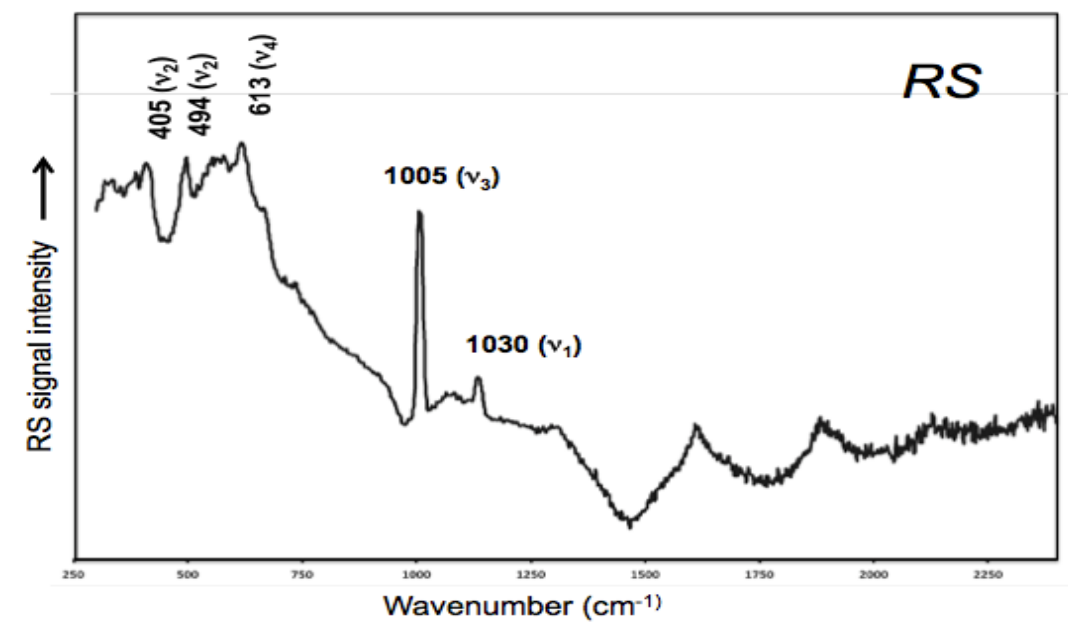

Figure 4. LIBS broadband spectrum for the playa soil from White Sands, NM, showing presence of $\mathrm{Ca}$ and $\mathrm{Sr}$ together with the minor presence of $\mathrm{Si}$ and $\mathrm{Na}$.

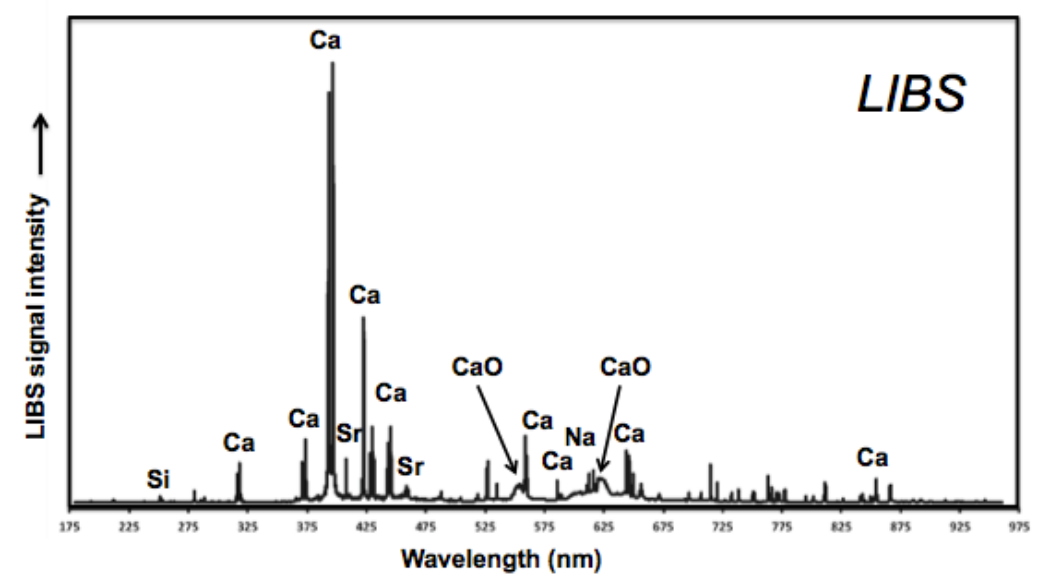




\section{Signal Processing, Statistical Analysis, and Data Fusion}

An investigation was undertaken of (i) different approaches to spectral data preprocessing and the fusion of the data outputs from current models of SciAps handheld analyzers for XRFS, RS, and LIBS and (ii) the use of advanced statistical analysis together with pattern recognition and machine learning for material classification and discrimination using the spectral data produced by these analyzers.

\subsection{Spectral data preprocessing}

LIBS analysis is complicated by the potential for variable interaction of the laser energy with the material being analyzed, so that the intensity of the light emission from the LIBS plasma can vary from laser shot to laser shot. Thus, LIBS spectral data needs to preprocessed so that only high-quality spectra are utilized for any data analysis application. Therefore, prior to the completion of the soil analysis effort, high quality LIBS datasets previously collected for other types of natural materials were used to assess the most effective method for data preprocessing. Preprocessing was also applied to the XRFS and RS data, as appropriate.

\subsubsection{Spectral truncation (XRFS) and normalization (XRFS, LIBS, RS)}

Due to the calibration process for XRFS, the spectra are often of slightly different lengths. Machine learning algorithms cannot process feature vectors of different length. Therefore, all XRFS spectra were truncated to ensure equal lengths. In order to ensure the classifier is not erroneously trained to make decisions based on relative intensity of XRFS, RS, and LIBS spectra, each intensity spectrum was normalized by subtracting the mean and dividing by the standard deviation.

\subsubsection{Argon spectral line removal (LIBS)}

The LIBS data collection process using an inert gas to enhance plasma intensity results in non-trivial emission line magnitudes at wavelengths of the Ar purge gas, regardless of the possible presence of Ar within the sample being interrogated. If these emission lines are constant or random across the identified spectral classes, then the partial least squares discriminant analysis (PLSDA) classifier should apply a zero or near-zero weight to the 
magnitudes of these lines during the training of the translation function that converts the raw data to a classification label. However, there is the potential that some bias will occur within these emission line magnitudes for any particular data set (especially those with limited samples) such that the classifier might interpret the Ar emission lines as informative features for classification and weight them more heavily. Therefore, it was considered prudent to remove these emission lines from the LIBS spectra to ensure classifier robustness.

As recorded, LIBS spectra contained emission lines for all wavelengths between 180 and $961 \mathrm{~nm}$. With Ar removal, only emission lines in the ranges of 190-675, 765-771, and 776-779 $\mathrm{nm}$ were retained. This process reduced LIBS spectra from 23,431 emission lines to 14,823 emission lines. The impact of argon removal was tested on several non-soil-based classification tasks, and no negative impact was observed.

\subsubsection{Baseline correction (LIBS)}

Although baseline drift was not observed to any great extent in the LIBS spectra for the soils analyzed during this project, substantial baseline drift was observed in some older LIBS data sets acquired with the same SciAps handheld analyzer. Thus, there is the potential that such variations in baseline level could confound the quality assessment of spectra (e.g. the calculation of signal-to-noise ratios) and negatively impact classification performance. Therefore, a baseline correction algorithm was developed that employs a 2-stage process that first removes the sparse peaks in the LIBS spectrum in order to estimate the baseline response, and then subtracts the estimated baseline from the original LIBS spectrum. In order to remove the peaks in the LIBS spectrum, a Hampel filter (Davies and Gather 1993) with a window size of $2 \%$ of the spectrum length was applied. For each sample, if the emission line magnitude was more than three standard deviations from the filter window's median, it was replaced by the median. Once the peaks were removed from the spectrum, the baseline of the spectrum was estimated by smoothing the residual with a median filter that was $0.5 \%$ of the spectrum length. This smoothed baseline estimate was then subtracted from the original spectrum. The baseline correction algorithm was added to the standard LIBS preprocessing workflow. 


\subsubsection{Spectra culling (LIBS)}

Some individual LIBS spectra appear to be anomalous outliers compared to the other spectra of a contemporaneously acquired set. Typically, such spectra correlate poorly with the other spectra collected from the same sample or they have a very low signal-to-noise ratio (SNR). It was hypothesized the removal of these outlier spectra would improve classification performance and, therefore, would be beneficial to add to the standard LIBS pre-processing procedure. Thus, the potential benefit of outlier spectra culling based on either SNR or low within-sample similarity was evaluated.

For evaluation, a sample-based decision-making approach was used, rather than spectra-based classification, since sample-based decision making is used by the sensor fusion algorithm and it has the potential to discount outlier spectra inherently. For sample-based decision making, all of the spectra confidences for a single sample are summarized by taking the average to give a single classifier label for a sample. By taking the average of the classifier confidences before making a final decision, the impact of outlier spectra may be potentially reduced (assuming that most spectra are not outliers), leading to a higher classification accuracy than might be observed if each individual spectrum is scored. The positive benefit of culling of non-standard (outlier) spectra was observed in LIBS dataset acquired for other types of geological materials and so was adopted for the soil data acquired during this project.

\subsubsection{Signal-to-Noise Ratio (SNR)}

As noted above, LIBS spectra demonstrate variability in quality that can impact classification performance. A robust, meaningful metric for estimating SNR is a tool needed for improving classification performance through poor data removal as well as a means of alerting users to the potential of system issues (e.g. if all data are of poor quality) in real time. In order to estimate SNR, the true signal without noise must be known. However, the true signal is rarely known under real-world conditions. Instead, an approximation of the true signal strength is estimated and used to calculate SNR.

Several potential methods of estimating the strength of the true signal in the LIBS spectra were compared in terms of computation load and perfor- 
mance. Performance was measured in terms of classification accuracy versus proportion of spectra culled. Ideally, a measure of SNR would maximize accuracy while minimizing the number of spectra culled, indicating that only the noisiest data were being discarded.

Two methods SNR estimation with low computation loads were evaluated: (i) the log of the peak spectrum value (Log-Max) and (ii) the ratio of the peak spectrum value to the median spectrum value (Peak-To-Median). Although the log of the peak spectrum value technically only estimates the signal strength, LIBS spectra with low signal strength tend to be spectra with low SNR. In addition to the two methods with low computation load, two methods with higher computation loads were considered. In the first method (Histogram-Split), an intensity histogram of the spectrum was generated, and between class variance was used to separate spectrum magnitudes into 'noise' and 'signal' values (Otsu 1979). Using these groups of magnitudes, the signal energy and noise variance were estimated, and SNR was calculated. In the second method (Gaussian-Outlier), a Hampel filter as described above for baseline correction was used to remove the peaks of the spectrum. In this method, the residual was used to estimate noise mean and variance. All spectrum magnitudes that were three standard deviations from the noise mean were considered part of the signal and used to estimate the signal energy. With the estimate of the signal energy and noise variance, the SNR was calculated.

Four methods were compared for several different LIBS data sets. Classification accuracy was estimated as increasing proportions of LIBS spectra were culled based on thresholding of the four metrics. For each metric, a limit was placed on culled spectra such that no sample's spectra were entirely discarded. The two low-computation-load methods were consistently among the best performers across data sets. The Histogram-Split approach tended to be a consistently poor performer and the GaussianOutlier method performed poorly for one of the data sets. Of the four SNR estimation metrics compared, the ratio of the normalized maximum spectrum value to the median spectrum value (Peak-to-Median method) most often achieved the greatest improvement in classification performance through spectra culling (removing spectra with lower SNR estimates). Also, the Peak-to-Median method most often achieved increases in accuracy more rapidly than the other methods. This indicates a better estimate of SNR than the other methods since the spectra that most impact classification performance are identified earlier (i.e. the worst spectra are given 
the lowest SNR estimates). The Peak-to-Median method was second only to one other method in terms of calculation speed, indicating viability for real- time use.

The potential benefit of spectra culling was tested on two geological data sets. Spectra were culled based on thresholding of either the SNR estimate or the similarity measure. The remaining spectra were classified, the confidences were averaged per sample, and a final label assigned based on the averaged confidences. This process was repeated for a range of thresholds ranging from no culling to the maximum number of spectra that could be culled without discarding any samples. Little benefit appeared to be gained from culling outlier spectra if no samples are discarded in their entirety. Rather, any potential benefit of evaluating spectra quality will likely be in terms of determining whether an entire sample should be remeasured. Given these results, this process was not added to the standard preprocessing workflow. However, there is potential for this method to be used as a tool to evaluate a data collection in real-time.

\subsubsection{Similarity}

Because LIBS is an analyzer that interrogates a sample at a spatial scale of 10 of microns, there always is the concern about sample heterogeneity affecting the LIBS analysis. This is a particular concern with the soil sample suite for this project, as the pellets analyzed were visually heterogeneous to variable extents. In addition to considering SNR as a method to cull outlier spectra, a second method of culling was considered based on the similarity between a spectrum and the other spectra collected for an individual sample.

To examine this effect, a spectral similarity analysis was undertaken. For this analysis, the pairwise spectral contrast angle was computed by treating each LIBS spectrum as a vector and calculating the angle between the two spectra (Krause et al. 1993). The spectral contrast angle can range from 0 to 1 , where $o$ indicates a perfect match. Figure 5 shows the estimated pairwise spectral similarities for a subset of 14 NIST and OREAS SRM soil standards. Each row represents a measurement site on the soil pellet that averages the full number of LIBS spectra collected at each site. Each column represents a similarity metric and the color-scale differences represent the value of the similarity metric. The yellow diagonal in each image is perfect similarity since this is the comparison of the spectrum 
with itself. Perfect similarity is one for correlation and zero for the similarity index and spectral contrast angle. Some of the 14 soil pellets show strong heterogeneity in this spectral variability analysis indicating textural heterogeneity within the pellet.

Figure 5. Spectral variability plot degree of homogeneity/heterogeneity between the different locations analyzed on soil pellets for 14 soil standards.

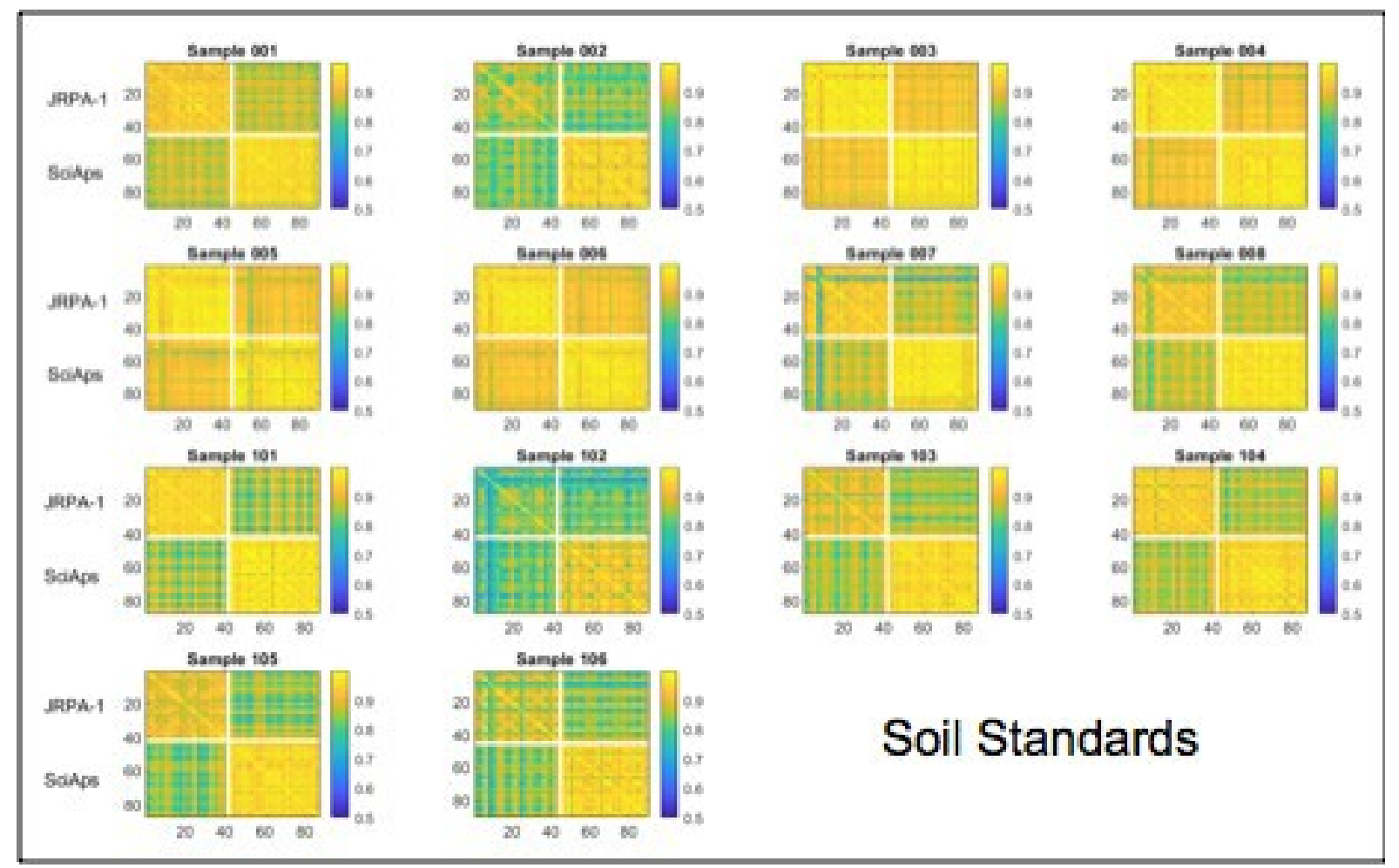

\subsubsection{Outlier rejection methodology}

A typical classification problem partitions the feature space such that every possible point in feature space is assigned to a class. However, in actual applications, it is likely that samples will be measured that are not members of any of the predefined classes. Rather than assign class membership to such samples, a better approach would be to alert the user of the system that these samples are likely "none of the above" (NOTA).

Outlier rejection requires a different approach to classifier design. Instead of setting a boundary between classes by finding the points that maximize the distance between the two classes, a boundary must be set around each individual class based on its data alone. Since there is no contrasting data to indicate where the boundary of one class ends and the 'outlier' class begins, a different approach must be taken. The approach used here is based 
on Hempstalk et al. (2008) that uses available spectra from each analyzer and class to estimate the mean and standard deviation at each wavelength. With this statistical model, a number of simulated spectra matching the number of measured spectra are generated. The outlier rejection, i.e. NOTA classifier, is trained to set a boundary between the measured spectra (not an outlier) and the simulated spectra (outliers). This process will set a boundary as tightly as possible to the measured spectra (Figure 6). The more accurate the statistical model, the tighter the outlier rejection boundary. Thus, this process requires a significant amount of measured data to ensure an accurate boundary.

Figure 6. Difference between typical classification (left) and outlier rejection (right).

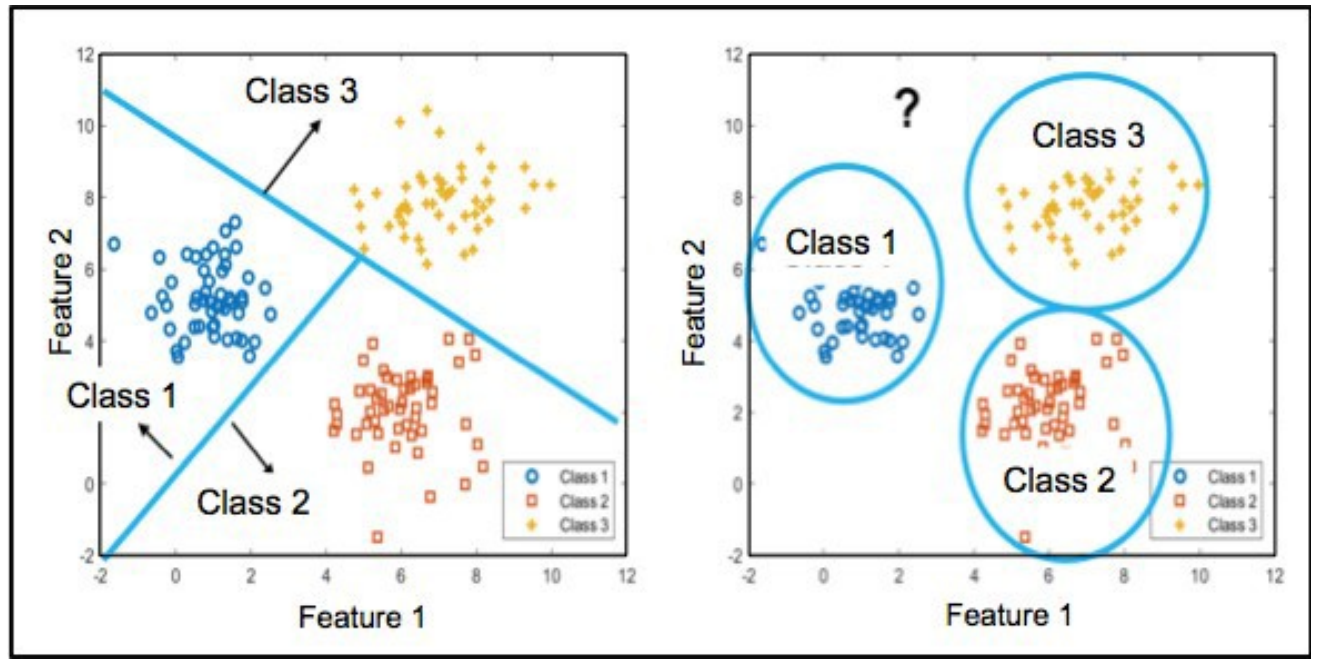

Figure 7. Implementation of the NOTA analytical process.

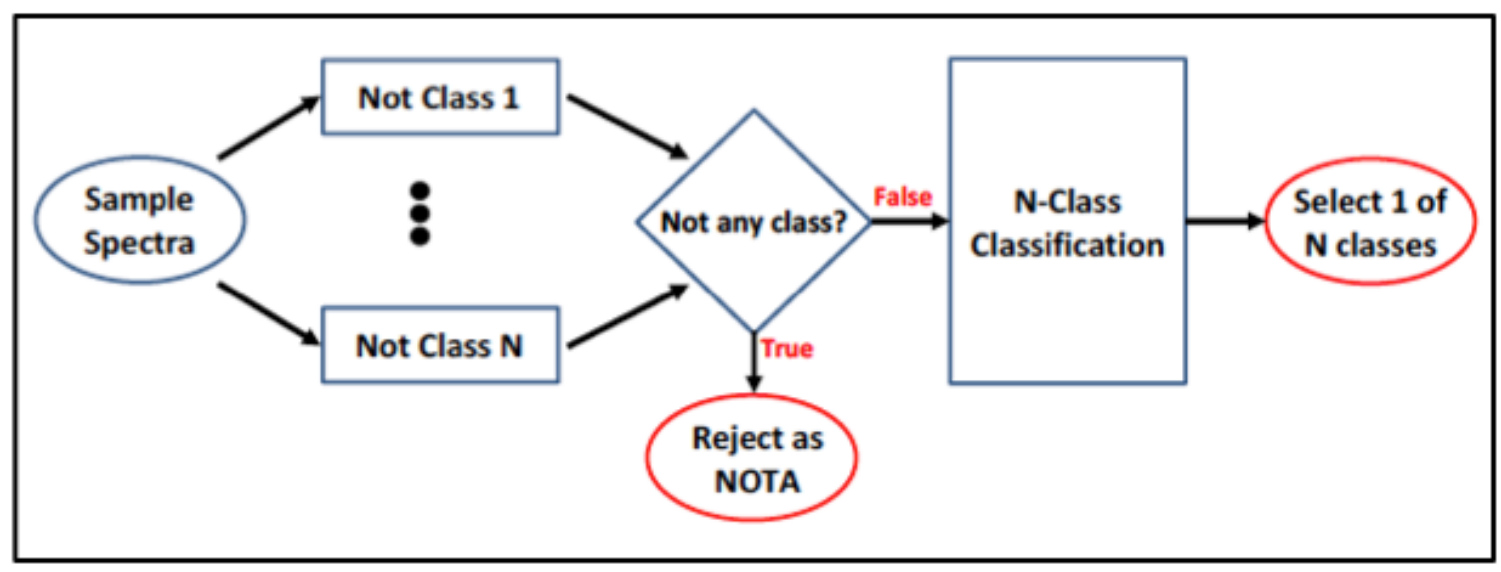


Rejecting "none of the above" (NOTA) samples is implemented hierarchically (Figure 7). A sample's spectra are tested against $\mathrm{N}$ binary "is/is not a class" classifiers. If all the sample's spectra are rejected by all the binary classifiers, then the sample is rejected as NOTA. If not, then the sample's spectra are passed on to the typically trained N-class classifier for determination of class.

Each NOTA classifier is a random forest classifier. Although Hempstalk et al. (2008) suggest that this process will work with any classifier type, results were only demonstrated with a tree-based classifier. Several types of classifiers were tested for this effort, and success was achieved only with the random forest classifier (a tree-based classifier).

In order to assess the NOTA classifier performance, two types of errors were considered: (i) NOTA samples that are not rejected, and (ii) nonNOTA samples that are rejected but would have been correctly classified if not rejected. Non-NOTA samples that would be incorrectly classified should arguably be rejected by the outlier rejection process. These are samples that do not fit the statistical models being used by the n-class classifier to assign a class label. Therefore, these samples were ignored during the NOTA classifier assessment.

\subsubsection{Outlier rejection results}

Two cases were considered. In the first, the non-NOTA data set was the New Mexico soil suite, and the NOTA data sets were the CSU soils, NIST standards, OREAS standards, and military installation soils. In the second, the non-NOTA data set was the military installation soil suite, and the NOTA data set consisted of the New Mexico soils, CSU soils, NIST standards, and the OREAS standards. Figure 8 shows an example of percent rejections for the different data sets as a function of the NOTA classifier operating points. The goal is to select an operating point that minimizes the rejection non-NOTA correctly classified samples while maximizing the rejection of NOTA samples. For example, selecting an operating point of 0.65 in Figure 8 would incorrectly reject approximately 9\% of correctly classified military installation soil samples while correctly rejecting 60$97 \%$ of the NOTA samples, depending on the NOTA data set. In addition, $75 \%$ of the incorrectly classified non- NOTA samples would be rejected by the outlier rejection classifiers. 
Figure 8. XRFS outlier rejection rates for different data sets when the non-NOTA data set is the military installation soil suite.

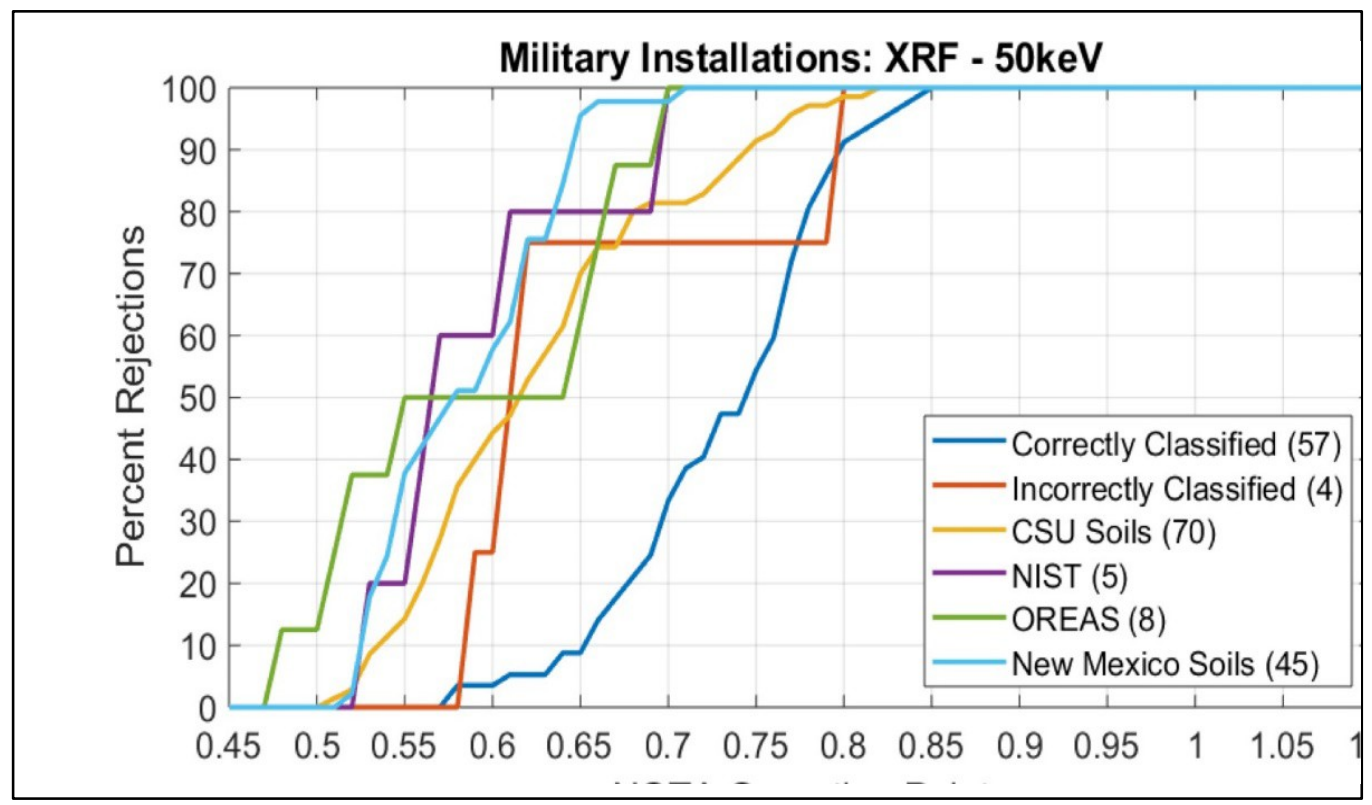

In Figure 9, the optimal operating point (assuming the cost of the two types of errors is equal) is selected for each test case and sensor and the proportion of rejections at that operating point is plotted. For the LIBS and XRFS data, between $70 \%$ and $100 \%$ of NOTA samples are rejected, depending on sensor and test case. Between $2 \%$ and $15 \%$ of non-NOTA samples were rejected. These results indicate the potential for outlier rejection with these sensors. While the approach was able to reject $60 \%$ of NOTA samples while rejecting $5 \%$ of non-NOTA samples for the New Mexico soils test case using the RS data, all non-NOTA samples were rejected before any NOTA samples for the military installation soils test case. This is likely due to the poor classification performance for the RS analyzer with the military installation soils. Since it is unable to determine an accurate model of the non-NOTA data, it is also unable to determine data that do not match those models. For the military installation soil RS data, it was not possible to reject any NOTA samples without rejecting all non-NOTA samples (hence the selected operating point to minimize error cost was a point that rejected no samples). 
Figure 9. Rejection rates at the optimal operating point for which the costs of the two types of errors (failing to reject NOTA samples and rejecting correctly classified non-

NOTA samples) were assumed equal.

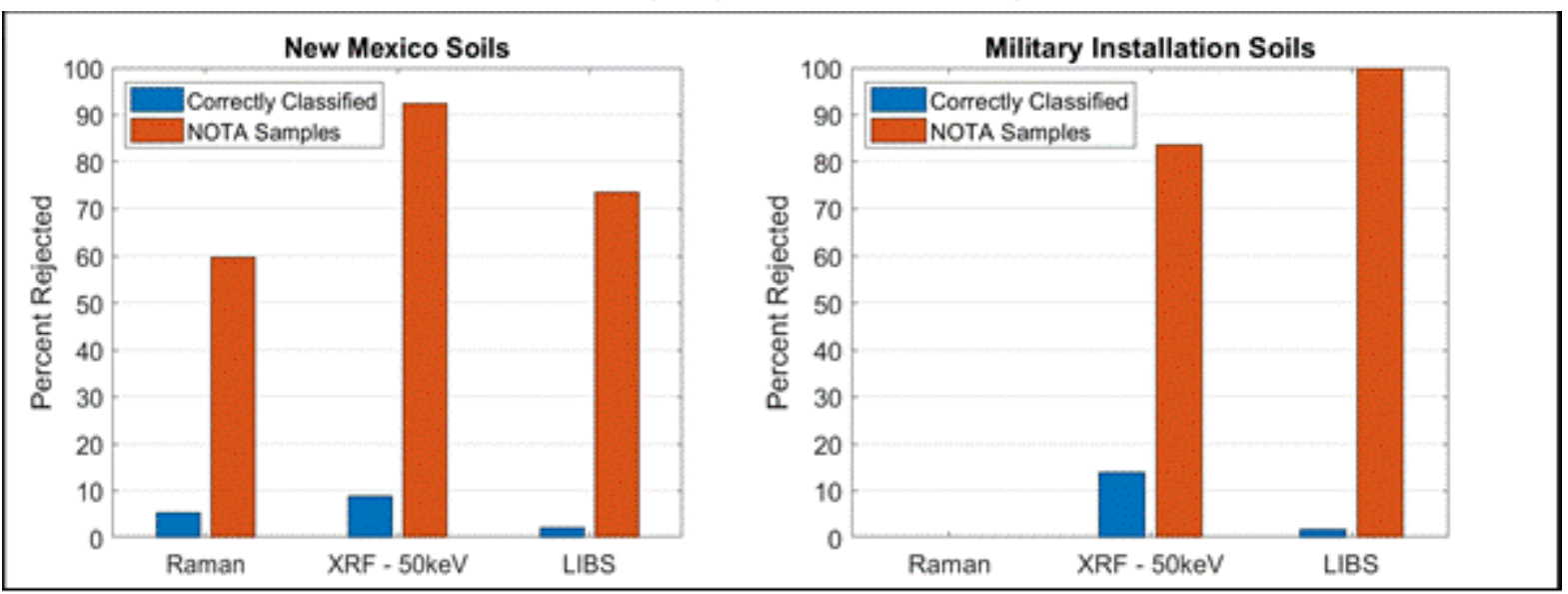

\subsection{Sensor information fusion}

In order to combine data for the multiple sensors, a hierarchical classification approach was used (Figure 10). For every sample, a set of $N s x D s$ spectra were collected for each sensor where $N s$ refers to the number of spectra for sensor $s$ and $D s$ refers to the dimensions of a spectrum collected by sensor $s$. The sensor-specific classifiers were applied to the spectra generating $N s x C$ classifier confidences that the sample's spectra belong to each of $C$ classes. In order to generate a feature vector for the sensor fusion classifier, the classifier confidences for each sensor were averaged to produce a single $1 x C$ vector. These vectors were then concatenated to create a single feature vector for the sample. This feature vector was classified by the sensor fusion classifier and a class label assigned to the sample based on the class that generated the highest fusion-classifier confidence. Partial least squares discriminant analysis (PLSDA) was used for both the sensor-specific and fusion classifiers, and classification accuracy was estimated using leave-one-sample-out cross-validation. 
Figure 10. Flow diagram for XRFS-RS-LIBS spectral fusion procedure.

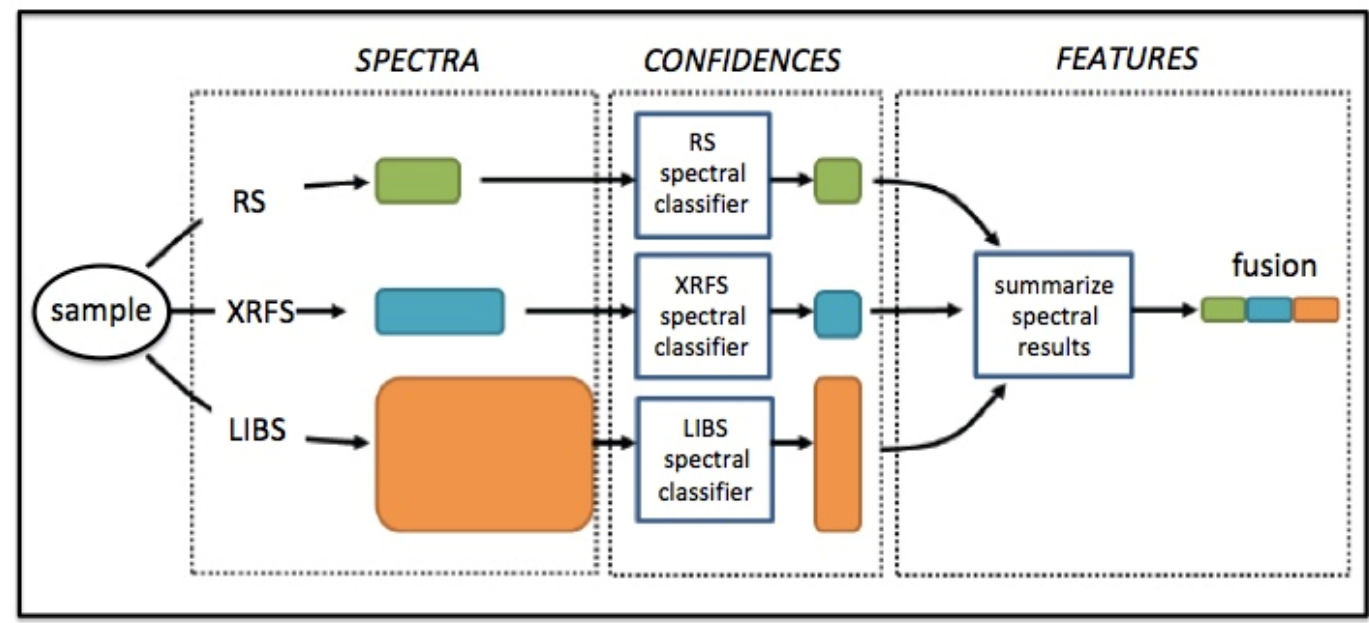

\subsection{Real-time signal processing}

One necessary step to progress from individual analyzers to a multi-analyzer system is real-time signal processing. Toward this end, a conceptual approach for real-time signal processing was developed. This approach, which as illustrated in Figure 11, consists of three modules - a file fetcher, a result monitor, and a sensor data processor. The software is expected to consist of three main modules that can be exchanged as needed (e.g. advancement in data acquisition). In addition, the data processing module will likely contain modules that can be exchanged to change operation (e.g. classifier, preprocessing). The file fetcher module handles all raw data interactions including polling the source directory for new files, ensuring files have been completely uploaded before transfer, transferring files to a 'process' directory to avoid interference issues with incoming data and previously recorded data, and reading the data out of files and into an appropriate format for processing. Once the file fetcher module has successfully transferred and converted the data files, the data processor performs all the analytics, and the result monitor displays the results for the user. The GUI allows the user to select their classification task and observe the likelihood that their sample is from each class as data are uploaded. Once the user has completed analysis of the sample, the user selects an archive button to send the data and a report of the results to a time-stamped folder. Processing is then cleared and ready for data from a new sample. 
Figure 11. Architecture diagram demonstrating expected software operation. The software consists of three main modules that can be exchanged as needed (e.g. advancement in data acquisition). In addition, the data processing module will likely contain modules that can be exchanged to change operation (e.g. classifier, preprocessing).
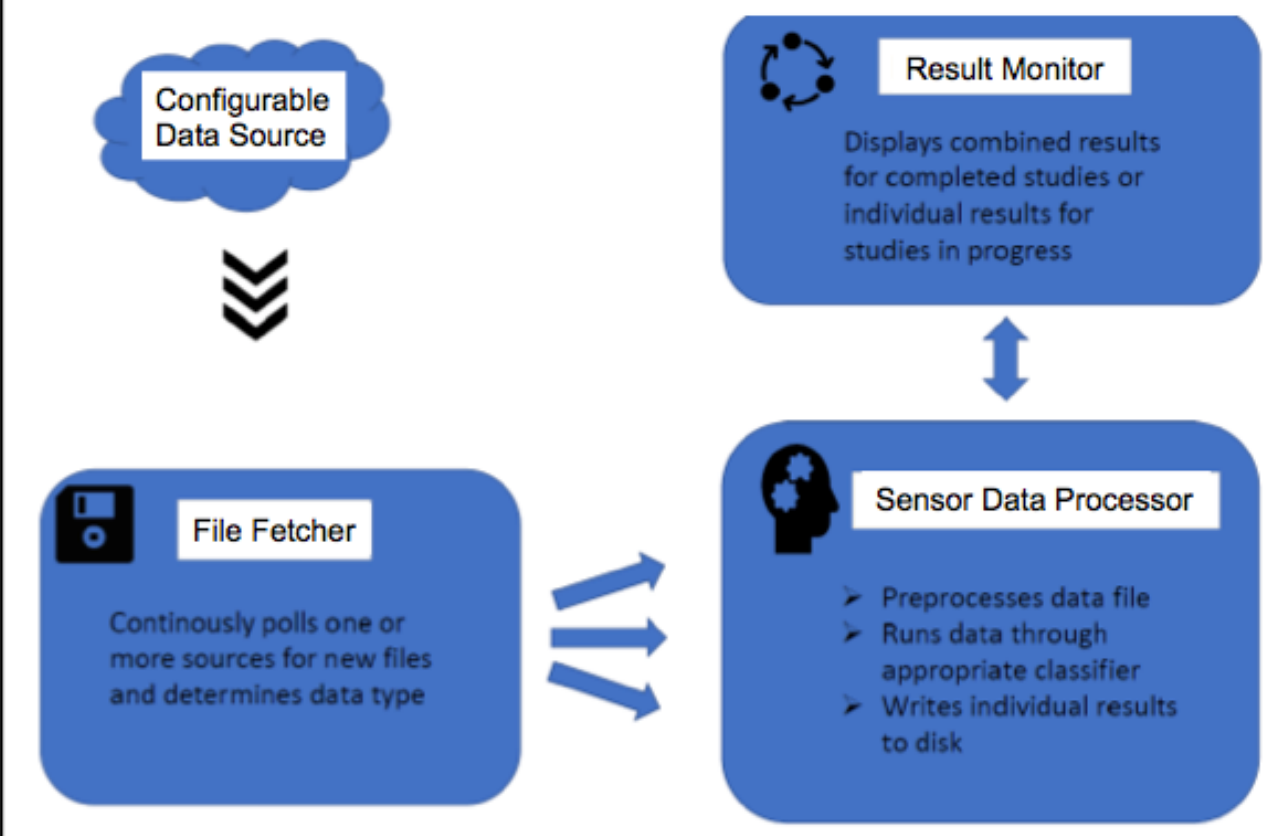

The software for real-time data processing developed for the project has the following features:

- The GUI allows the user to select the appropriate classifiers;

- The GUI allows the user to select the classes to be discriminated;

- The GUI allows the user to select the sensors to be used for fusion;

- The GUI monitors the underlying modules to alert the user if a problem arises;

- As data files from the sensors are uploaded, the GUI will display confidence that the sample is from each class for each sensor for which there are data;

- The GUI automatically polls the input directory and updates the display as new files are added;

- Once the user is satisfied with the result, they can select to archive the data which will move the data to a storage location and include a report

- The archive button clears the display, creates a date-stamped directory for storage, and moves the sensor files to the new directory with a classification report. 
This capability was field tested during in August 2019, when the data streams from the three analyzers were in real time wirelessly transmitted during analysis to a laptop computer.

During the project, three reference cases were added to the software for real-time estimation of class confidences for any unknown sample: New Mexico soils, military installation soils, and New Hampshire soils. For the latter, only LIBS processing is possible due to the limited data collection. For the first two, individual data processing was possible for XRFS, RS, and LIBS data, as well as data fusion processing data from all three analyzers. At the conclusion of the project, the software polls an input directory into which the user places sensor spectra CSV files. The software automatically determines the analyzer type of the new data, processes the data with the appropriate spectra classifier, and displays a confidence that the sample belongs to each of the three classes in the library. Once data from all three analyzers has been added, the spectra classifier outputs are fused and confidence that the sample belongs to each class is displayed. Once the user has completed analysis of the sample, the user selects an archive button to send the data and a report of the results to a time-stamped folder. Processing is then cleared and ready for data from a new sample. 


\section{Data Analysis and Spectral Fusion Results and Discussion}

\subsection{Spectra data preprocessing}

All spectra from the three handheld analyzers were individually normalized using z-scoring (mean subtraction and standard deviation division). Characterization features and XRFS quantitative features were normalized across samples using z-scoring (cross- validation normalization was used during classification). In addition, for the three analyzers, the following preprocessing was applied - RS: No preprocessing; XRFS: Spectra were truncated to be the same length; LIBS: All spectra were baseline corrected and had Ar spectral peaks removed.

\subsection{New Mexico Soil Suite}

Soils were sampled from six locations across central-north New Mexico in November and December 2018. Six samples were collected from each location along a straight transect approximately $22 \mathrm{~m}$ apart, taking about 500 $\mathrm{g}$ from the top $\mathrm{o}-5 \mathrm{~cm}$ of the soil. The six soils sampled for the project (Table 2) were (i) the loamy fine sand of the Bluepoint Soil Series at the Rio Salado, (ii) the very stony sandy loam of the Laborcita-Pilabo-Lemitar Soil Series Complex in Upper Socorro Canyon, (iii) the very stony sandy loam of the Laborcita-Pilabo-Lemitar Soil Series complex in Lower Socorro Canyon, (iv) the gravelly sandy loam of the Millett-Sedillo Soil Series complex at Sedillo Hill, (v) the gypsiferous sand of the Lark-Transformer Soil Series association at White Sands, and (vi) the strongly saline silty clay loam of the playa soil at Willard. At Rio Salado, two sets of samples were gathered: one at depth $0-3 \mathrm{~cm}$ and one at $10-13 \mathrm{~cm}$.

Seven sets of six samples were collected to determine if the multi-analyzer approach could distinguish different soils and identify similar soils. The two sets at Rio Salado contain the same soil to test whether these samples would be identified as similar. All other soils are different one from the other and from the Rio Salado soil to test whether these would be identified as different soils. Three soils differ only by age: Sedillo Hill (740,00o years), Socorro Canyon Upper (140,000 years), and Socorro Canyon Lower (25,000 years). These soils were sampled to test whether soils that are different in some respects (i.e. age) but are similar in other aspects (i.e. 
parent material, climate, flora and fauna, and topography) could be discriminated. These samples were analyzed by JRPA in January, March, August, and December 2019 to evaluate the benefits of sensor fusion performance through multiple analysis of the same sample suite.

The 5-class schema for New Mexico soils was used - Rio Salada, Sedillo Hill, Socorro Canyon Upper/Lower, White Sands, and Willard. The six soil pellets from each locality were measured on four collection dates (December 2018, January 2019, March/April 2019, and August 2019). Spectral fusion was first undertaken for the January 2019 data collection using the spectral data from the three analyzers (XRFS, RS, and LIBS), first individually, then LIBS with XRFS, and finally the spectral data for all three analyzers.

The result of this fusion exercise is shown in Figure 12, where the classification accuracy for RS is seen to approach $80 \%$, that for LIBS better than $85 \%$, and that for XRFS almost 90\% for the January 2019 data collection. So, it is not surprising that the accuracy for 2- or 3-analyzer fusion was better than 90\%. For the March/April 2019 data collection, this diminished to just under $75 \%$ for RS, but increased to better than $95 \%$ for both LIBS and XRFS. By comparison, accuracy for dual analyzer fusion involving RS was lower than for January 2019, that for LIBS plus XRFS was improved, and that for the fusion of spectral data for all three sensors was marginally only diminished. Fusion of the results from both dates exhibited full classification accuracy for the 3-analyzer fusion. 
Figure 12. Classification accuracy for the New Mexico soil suite based on consideration of spectra for XRFS, RS, and LIBS; fusion of spectra for RS + XRFS, LIBS + XRFS; and for RS + LIBS + XRFS for spectral data collected in Jan 2019, Mar/Apr 2019, and for data fusion from both dates.

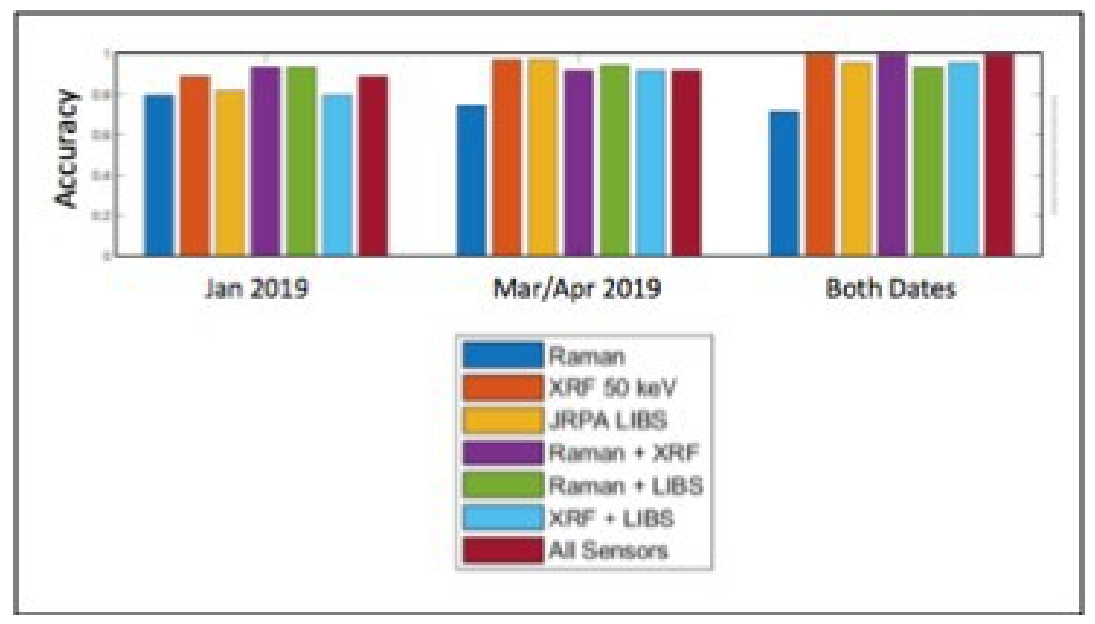

In addition, two LIBS systems were used in each of these data collections. The number of samples per class varied per data collection (Table 3) with an incomplete set of samples analyzed in December 2018 and August 2019, so that cross-validation analysis for these collection dates is not possible.

Table 3. Number of samples per class for each collection date.

\begin{tabular}{|l|c|c|c|c|}
\hline & Dec 2018 & Jan 2019 & Mar 2019 & $\begin{array}{c}\text { Aug 2019 } \\
\text { (*LIBS SciAps) }\end{array}$ \\
\hline Rio Salada & 2 & 14 & 6 & 12 \\
\hline Sedillo Hill & 2 & 8 & 6 & $6^{*}$ \\
\hline Socorro Canyon & & 11 & 11 & $12^{*}$ \\
\hline White Sands & & 6 & 6 & 6 \\
\hline Willard & & 6 & 6 & $6^{*}$ \\
\hline
\end{tabular}

Data analysis early in the project suggested that training and testing on separate collection dates resulted in poor classification performance for LIBS data. This observation raised the following questions:

1. Do the other analyzers experience the same decrease in performance across collection dates?

2. Can the impact of collection date be mitigated by pooling multiple collection dates worth of data for training? The hypothesis is that 
pooling multiple collection dates will result in more robust class models.

3. How is sensor fusion impacted by collection date?

Leave-one-sample-out (LOSO) cross-validation was used to assess the collection dates for which multiple samples had been measured for all classes. In addition, cross-validation was used to assess data pooled across all collection dates. Classifiers were trained for each collection date for which multiple samples per class were available. These classifiers were used to classify data from non-matching collection dates regardless of the number of samples per class in the test set. Data were pooled across all but one collection date and used to train a classifier that was then used to classify data from the remaining collection date, e.g. the Dec 2018, Jan 2019, and Mar/Apr 2019.

Figure 13. Single analyzer classification results for three different methods of training.
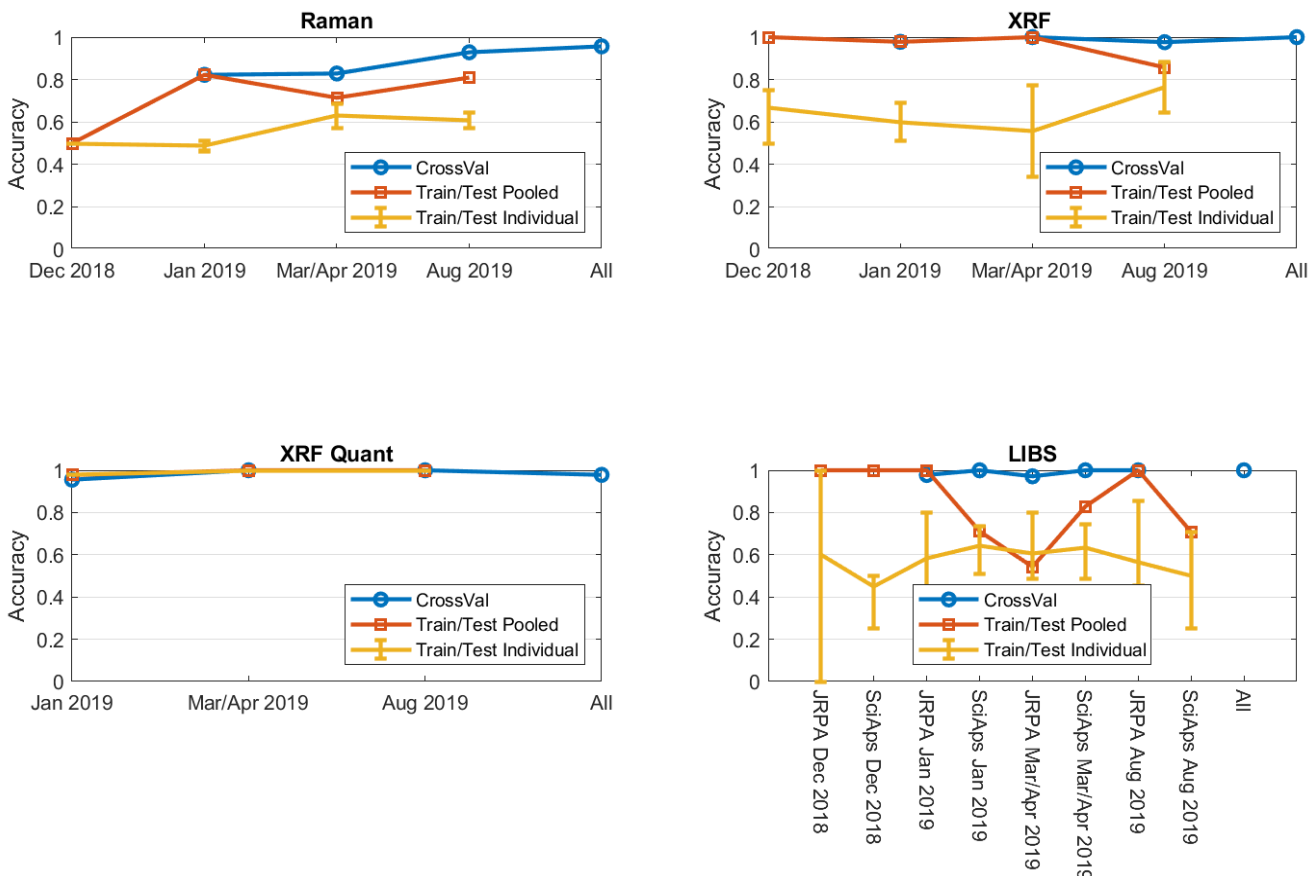

Single analyzer results are shown in Figure 13. Cross-validation and pooled collection dates result in a single estimate of accuracy. For the individual collection date train/test classification, the results are plotted as an average with the error bars indicating the cases with the highest and lowest accuracy (i.e. the full range of accuracy observed). For example, for the RS analyzer, cross-validation for the Jan 2019, Mar/Apr 2019, and Aug 
2019 collection dates ranged from $82 \%$ to $93 \%$ correct. Pooling the data across all collection dates and using cross-validation resulted in $95 \%$ correct accuracy. There are no cross-validation results for Dec 2018 since the data set is incomplete. Testing the Jan 2019 data against classifiers trained with the Mar/Apr2019 or Aug 2019 data sets results in an average accuracy of $49 \%$ correct, with little

If, however, the Jan 2019 data are classified using a classifier trained on both the Mar/Apr 2019 and Aug 2019 data sets, accuracy increases to the cross-validation accuracy of $82 \%$ correct. The classification based the quantitative XRFS analyses is thoroughly robust to collection date, whereas results from all three analyzers are all negatively impacted by training and testing across collection dates. Pooling across collection dates mitigates this issue to varying degrees. The same methods of classification accuracy estimation were used for the multiple sensor analysis as for the single-sensor test (cross-validation, train/test across individual dates of collection, and train/test with pooled collection dates). Two fusion cases were considered: (i) RS + XRFS + LIBS and (ii) XRFS + LIBS. The spectral data acquired by the two LIBS analyzers (JRPA and SciAps) were kept separate such that each collection date resulted in two sets of accuracy results.

Figure 14 shows results for fusing XRFS and LIBS sensor data. Cross-validation results are consistently near $100 \%$ correct. As with the individual sensors, training with pooled data tends to result in higher accuracy than training with an individual collection date. Figure 15 shows results for fusing XRFS, RS, and LIBS data. Results are similar to those for the singleanalyzer results for just XRFS or LIBS. Ignoring the Dec 2018 collection date, accuracy for the 3 -analyzer fusion with pooled collection date training appears to be marginally more robust across collection dates than for the XRFS and LIBS sensor fusion. It is important to note that each collection date has a small number of samples, so it is possible that the impact of pooling is not just due to capturing variability across dates of collection. It might also be due to the increase in the overall amount of information. 
Figure 14. Classification results for XRFS and LIBS fusion for three different methods of training. For each data collection, XRFS data is paired with that from either the JRPA LIBS analyzer or the SciAps LIBS analyzer.

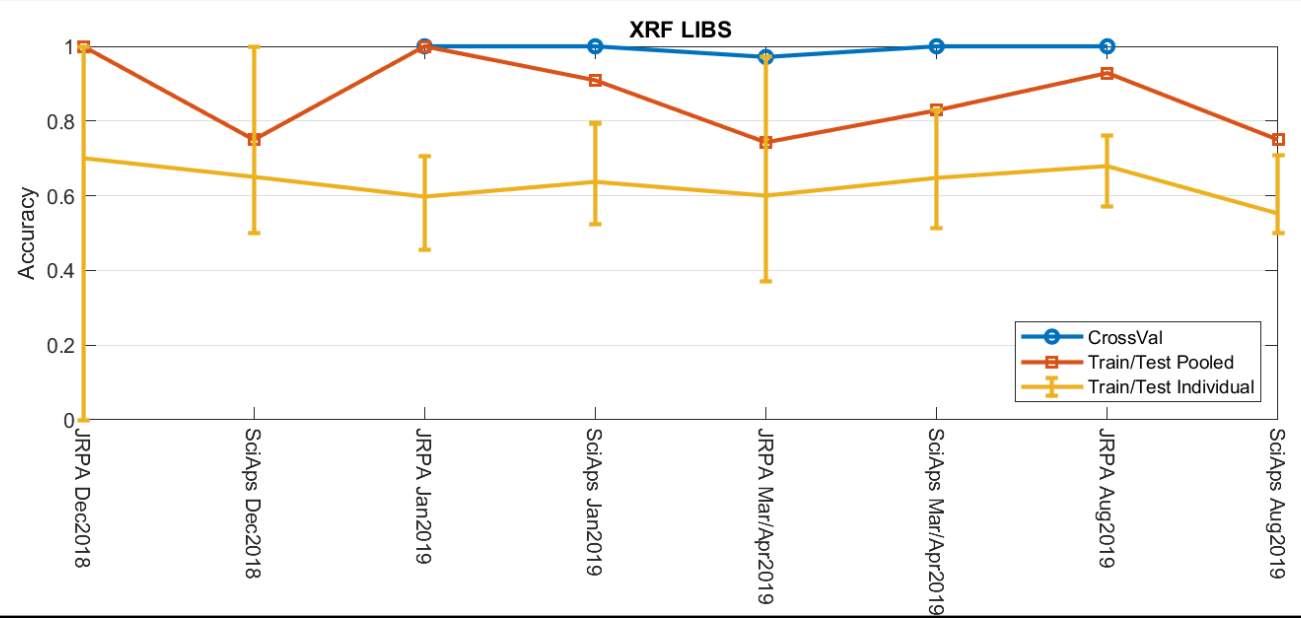

Figure 15. Classification results for fusing the Raman, XRFS, and LIBS sensors using three different methods of training. For each data collection, Raman and XRF are paired with either the JRPA LIBS sensor or the SciAps LIBS sensor.

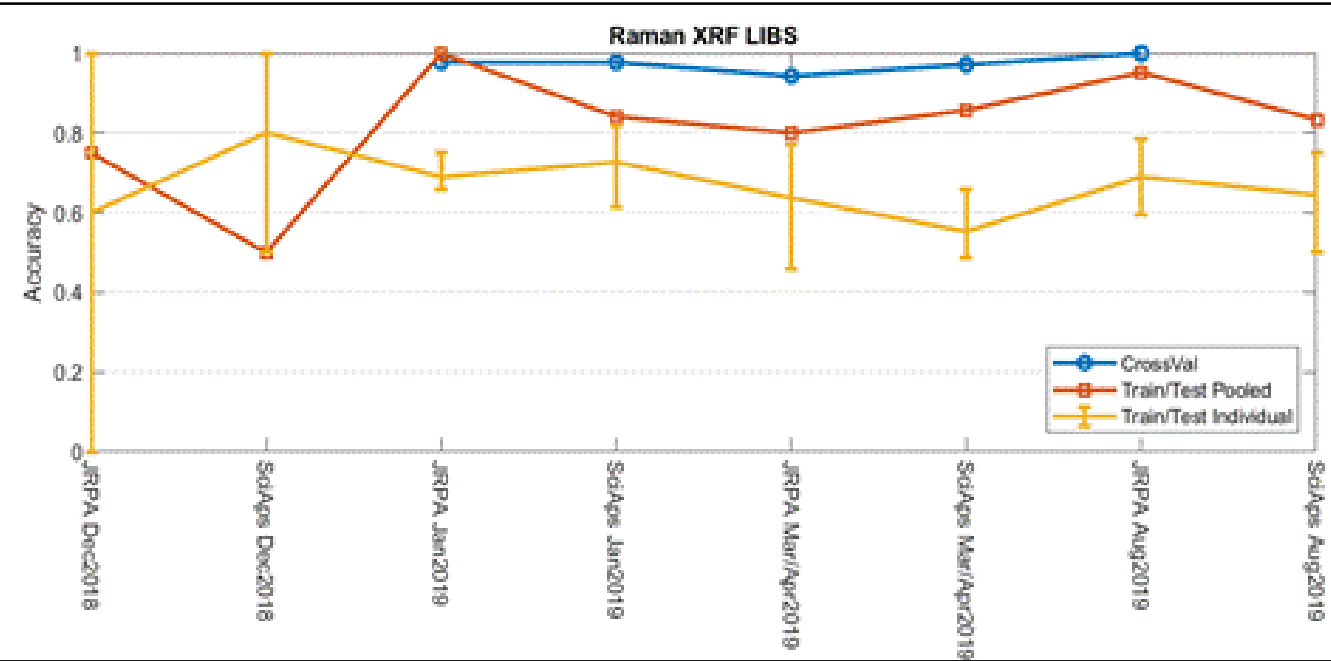

All the spectral measurements appear susceptible to decreased accuracy when training/testing across collection dates, whilst the XRFS quantitative data was not susceptible. Pooling data did mitigate the impact of collection date to some degree; however, it is important to note that this may be due to the increase in the amount of information as much as it is due to better capturing variability across collection dates. Pooling data is the equivalent of measuring a single sample in nine locations instead of three for LIBS or measuring a sample nine times instead of three times for XRFS. This may be important when there are only a small number of samples per class. 
Thus, measuring a single sample in multiple locations is likely beneficial although not as much as the measurement of more samples.

\subsection{Military Installation Soil Suite}

A second suite of 58 soil samples came from six U.S. military installations (Ft. Wainwright, AK; Ft Lewis, WA, Idaho National Guard Camp Kimama; Ft. Benning, GA; Ft. Eustis, VA; and Massachusetts Military Reservation/Joint Base Cape Cod). Provided by Dr. J. Clausen of the USACEERDC CRREL, these soils were collected from the top $2.5 \mathrm{~cm}$ of soil at small arms firing ranges and, therefore, are contaminated to various extents with metal elements present in munitions (such as $\mathrm{Cu}, \mathrm{Zn}, \mathrm{Pb}, \mathrm{W}$, and $\mathrm{Sb}$ ). A range of soil compositions, varying from uncontaminated to strongly contaminated, were analyzed for each of the six military installations. The NRCS Soil Series to which these soils are assigned is not known. These six military installation soil suites were processed during the March- April 2019 period of analytical work.

Figure 16. Classification accuracy for the military installation soils based on RS, XRFS, and LIBS data; fused 2-analyzer data, and fused 3-analyzer data.

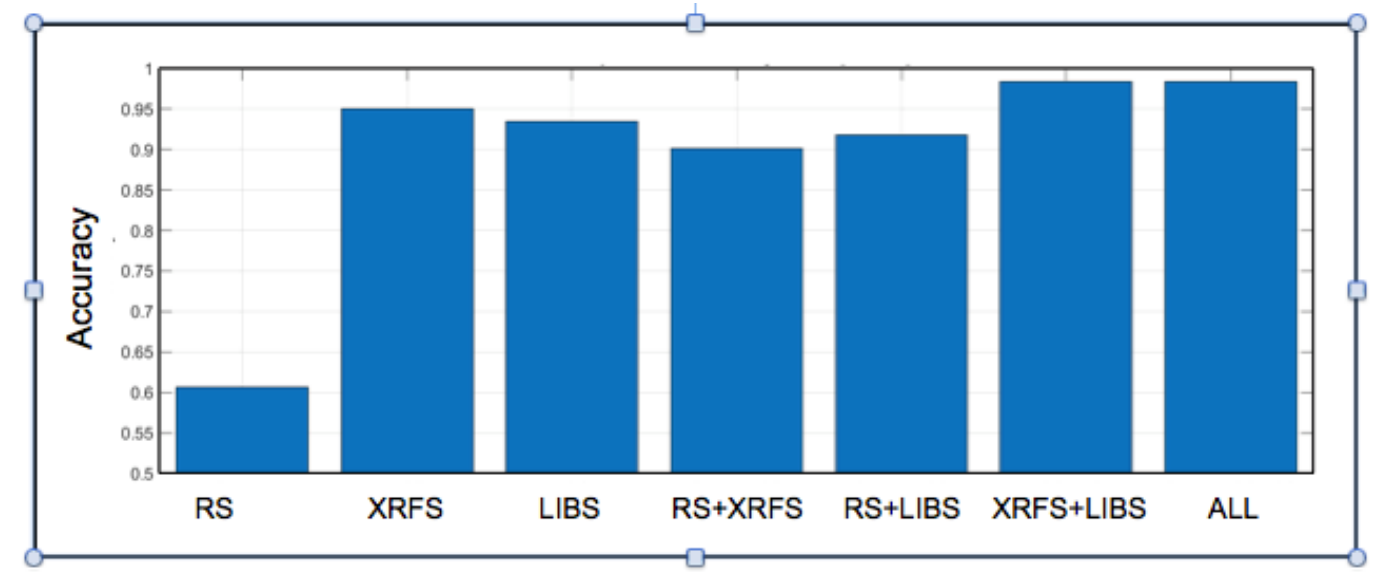

A single data for sets for the sets of 10 surface soils at small arms ranges on six military installations were acquired in Mar/Apr 2019. A 6-class classification schema was used with the following classes: MMR-Joint Cape Cod (MA), Ft. Eustis (VA), Ft. Benning (GA), ING Camp Kimama (ID), Ft. Lewis (WA), and Ft. Wainwright (AK). The result of this fusion exercise is shown in Figure 16, where the classification accuracy for RS is around $60 \%$, and that for XRFS and LIBS $>90 \%$. Thus, the fusion results for XRFS + LIBS and for all analyzers exhibit a high accuracy that is $>95 \%$. 


\subsection{CSU Agriculture Soil Suite}

Through an arrangement with Dr. Robert Miller of the Department of Crop and Soil Science at Colorado State University (CSU) a collection of 166 agricultural soils were provided to the project that were selected from the ALP Laboratory Proficiency Program (ALP-LPP) archive, of which 144 were analyzed during this project. The soils of the ALP-LPP program were collected across 32 US states and 7 Canadian provinces. The samples received, which had been milled to $<0.7 \mathrm{~mm}$ and homogenized, were from the top $20 \mathrm{~cm}$ of the soil profile at the selected sampling locations. The U.S. soils, which are from all 12 of the NRCS soil orders, are particularly well characterized compositionally through analytical work undertaken by the ALP-LPP program, with quantitative chemical information available for $>35$ specific parameters of interest to the agricultural soil science community. Analysis of the first 96 samples of this suite was undertaken in January 2019, with a subsequent 48 samples analyzed in August 2019. These 144 soils represent 10 of the 12 NRCS Soil Orders, but three of the classes are only represented by a single sample and another only has two samples.

There is no single classification schema for the CSU soil suite, which can be classified in multiple different ways. Therefore, it was decided to examine other descriptive features ascertainable from the Soil Series descriptions for each soil (i.e. soil thermal regime, soil moisture regime, geologi$\mathrm{cal} /$ mineralogical character, and geological source) in the context of the five soil-forming factors (climate, parent material, landscape setting, biota, and time) to see if any of these influences could be teased out from the statistical analysis. For purposes of the statistical analysis, analytical data for the CSU sample suite was grouped for processing according to a 5class schema - NRCS Soil Order, thermal regime, moisture regime, mineralogical character, and geological source (Table 4). The feature sets used were spectra from the individual XRFS, RS, and LIBS analyzers, the quantitative XRF analyses, the laboratory chemical analyses for the samples, and a combination of all features. Results are shown in Figure 17 and the classification matrices for the random forest classifier with text features are shown in Figure 18. For the sensor fusion, samples were discarded that were not measured using all the handheld analyzers and/or did not have a full suite of analytical metadata. This resulted in the loss of a few samples from the processed dataset. 
Table 4. Classification schema for CSU soil suite characterization.

\begin{tabular}{|c|lc|}
\hline \multirow{2}{*}{ SCHEMA } & CLASS & \# SAMPLES \\
\hline \multirow{4}{*}{ Soil Order } & Alfisol: & 16 \\
& Aridsol: & 5 \\
& Entisol: & 10 \\
& Inceptisol: & 10 \\
& Mollisol: & 40 \\
& Ultisol: & 8 \\
\hline \multirow{3}{*}{ Thermal } & Frigid: & 15 \\
Regime & Mesic: & 54 \\
& Thermic: & 18 \\
& Hyperthermic: & 3 \\
\hline \multirow{4}{*}{ Moisture } & Aquic: & 12 \\
Regime & Aridic: & 6 \\
& Udic: & 50 \\
& Ustic: & 13 \\
& Xeric: & 10 \\
\hline \multirow{3}{*}{ Mineralogical. } & Calcareous: & 14 \\
Sharacteristic. & Kaolinitic: & 4 \\
& Siliceous: & 5 \\
& Smectitic: & 7 \\
\hline \multirow{3}{*}{ Geological } & Alluvium: & 10 \\
Source & Crystalline Rock: & 17 \\
& Glacial Material: & 20 \\
& Loess: & 16 \\
& Marine Sediment: 14 \\
\hline
\end{tabular}

Toward this end, the spectral data from the three analyzers, first individually, next fused together 2x2, then fused all together, and finally all information about the samples (i.e. spectral data, XRFS quantitative analyses, and laboratory chemical analyses) fused together. As illustrated in Figure 17, a moderate level of success was realized when classifying soils based on soil thermal regime, soil moisture regime, and geological source. This result is not surprising given that only one sample was available per soil, when what was really needed for robust statistical analysis was 5-10 separate samples of each soil. 
Figure 17. Classification accuracy for the CSU classification schema based on consideration of spectra for Raman spectroscopy - RS (1), X-ray fluorescence spectroscopy - XRFS (2), laser-induced breakdown spectroscopy - LIBS (3), fused spectra for RS + XRFS (4), RS + LIBS (5), and XRFS + LIBS (6), RS + XRFS + LIBS spectra (7), fused spectra + XRF quantitative analyses (8), and fused spectra + XRF quantitative analyses + laboratory chemical analyses (9).

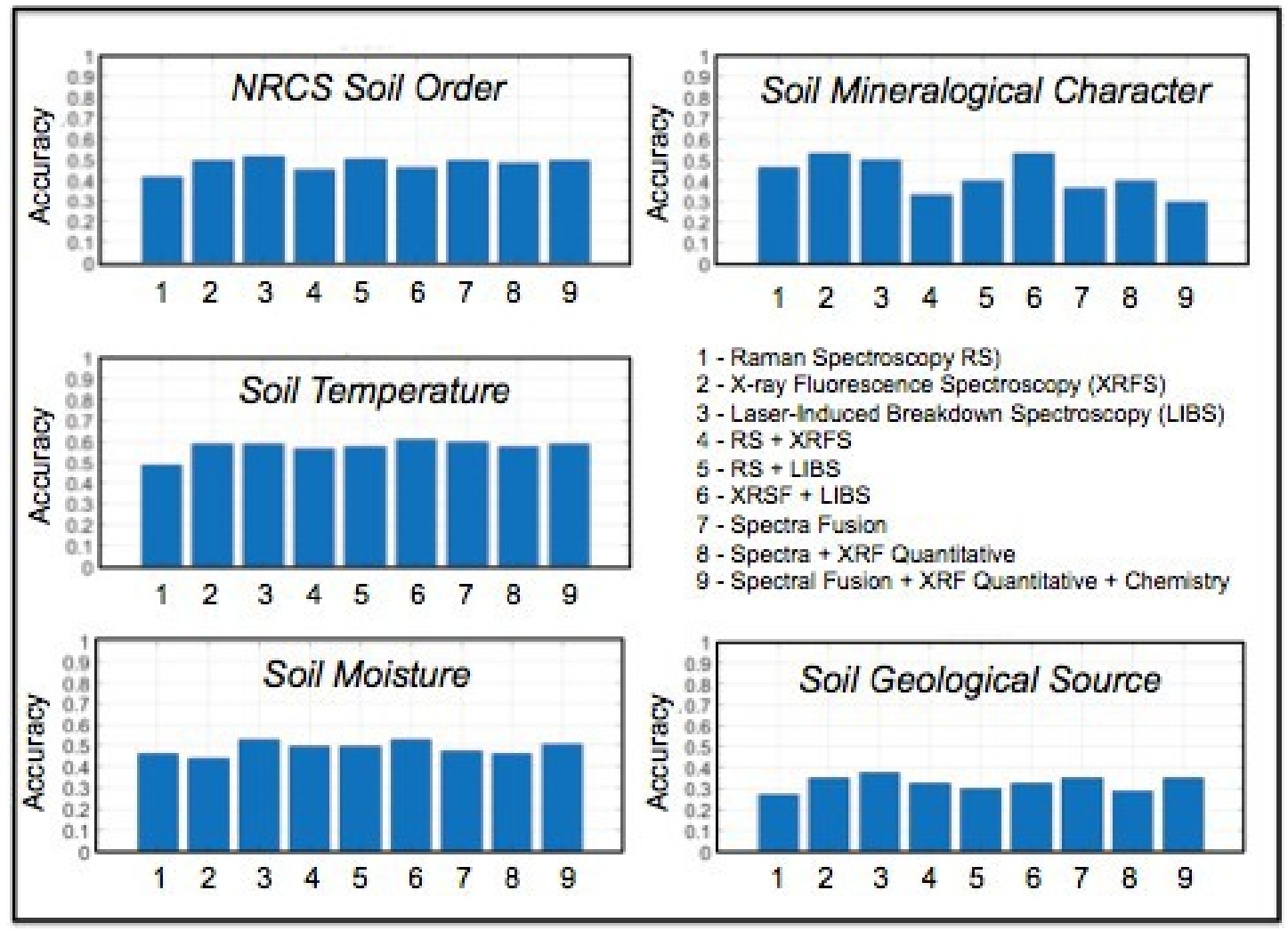

To further investigate the situation, classification accuracy with each individual characterization feature was calculated in order to assess the relative information content of each feature. The results for two classifiers (PLSDA and random forest) are shown in Figure 18. These plots indicate some features in each schema may provide no information for the classification. The legends include the accuracy expected from always selecting the class that has the most samples using the 'majority rule' method for evaluating the performance of a classifier when the classes are imbalanced. Also, many features appear similarly informative on their own. Although not necessarily indicative of their behavior in combination with other features, it does suggest a potential issue for consistent feature selection. When multiple features provide similar information content, the search space for the best selection of features can contain many local performance maxima that can confound the search for the true global maximum. 
Figure 18. Independent feature accuracy per CSU soil classification schema. Features are sorted on a per schema basis, greatest accuracy to least accuracy. The legends indicate classification performance that could be achieved by always selecting the class with the most samples.

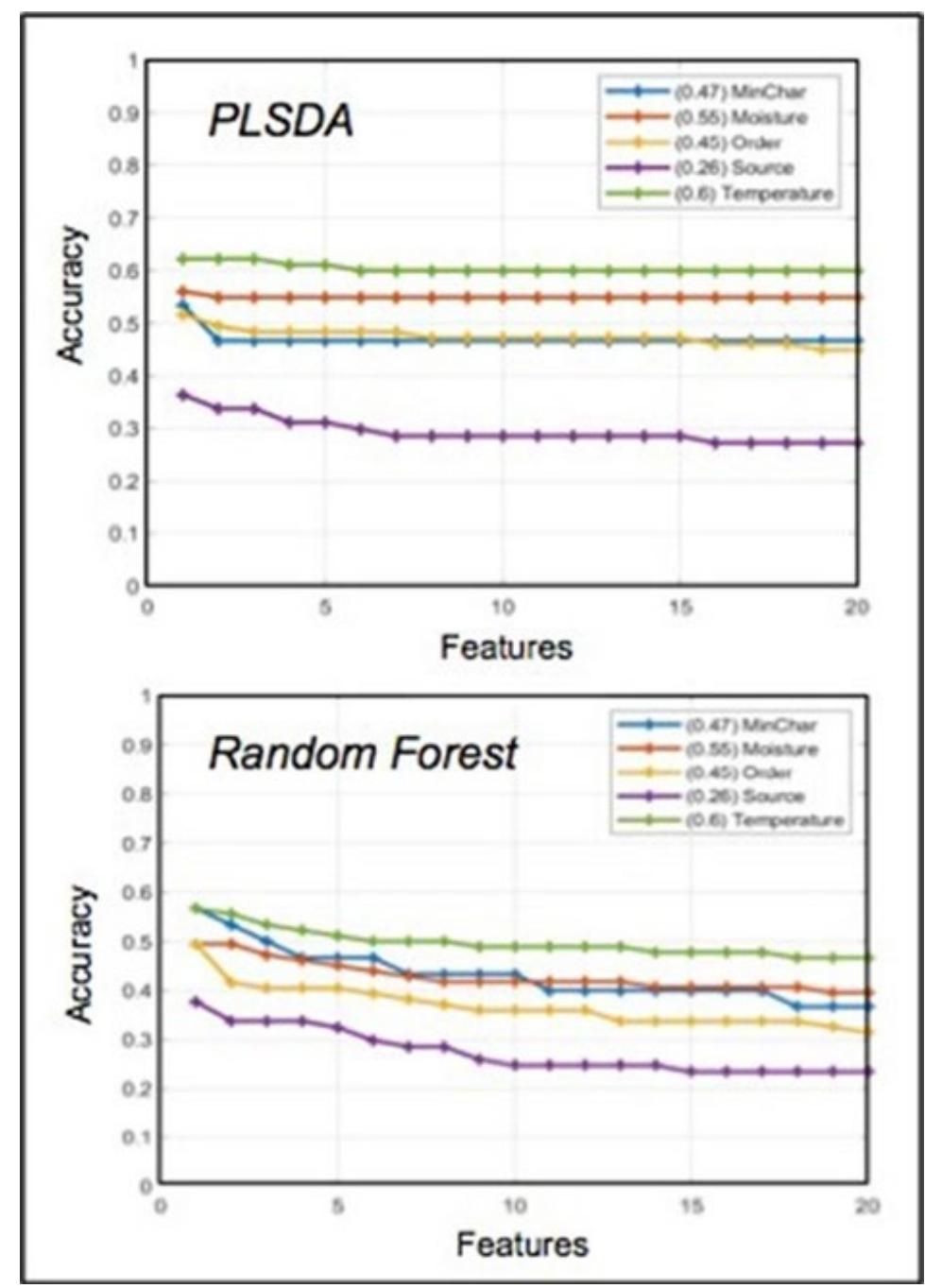

To further investigate the situation, classification accuracy with each individual characterization feature was calculated in order to assess the relative information content of each feature. The results for two classifiers (PLSDA and random forest) are shown in Figure 18. These plots indicate some features in each schema may provide no information for the classification. The legends include the accuracy expected from always selecting the class that has the most samples using the 'majority rule' method for evaluating the performance of a classifier when the classes are imbalanced. Also, many features appear similarly informative on their own. Although not necessarily indicative of their behavior in combination with other features, it does suggest a potential issue for consistent feature selection. When multiple features provide similar information content, the search 
space for the best selection of features can contain many local performance maxima that can confound the search for the true global maximum.

Finally, principal components analysis was conducted on the CSU soil suite for Soil Series represented by two or more individual samples. Figure 19 presents PC scores plots for XRFS, RS, LIBS, and chemical composition determined through laboratory analysis. It is notable that samples from the same Soil Series tend to be closely associated in PC space in Figure 19. Whereas, class discrimination for samples of the CSU soil suite according to NRCS Soil Order, soil temperature or soil moisture regime, or soil mineralogical character or geological source was not successful (Figure 18), the PC scores plots of Figure 19 suggest Soil Series discrimination might be possible based on analysis of a larger set of samples from each Soil Series.

Figure 19. Principal component analysis score plots for XRFS (upper left), LIBS (lower left), RS (upper right), and chemistry (lower right) for the soil series of CSU soil suite represented by multiple samples
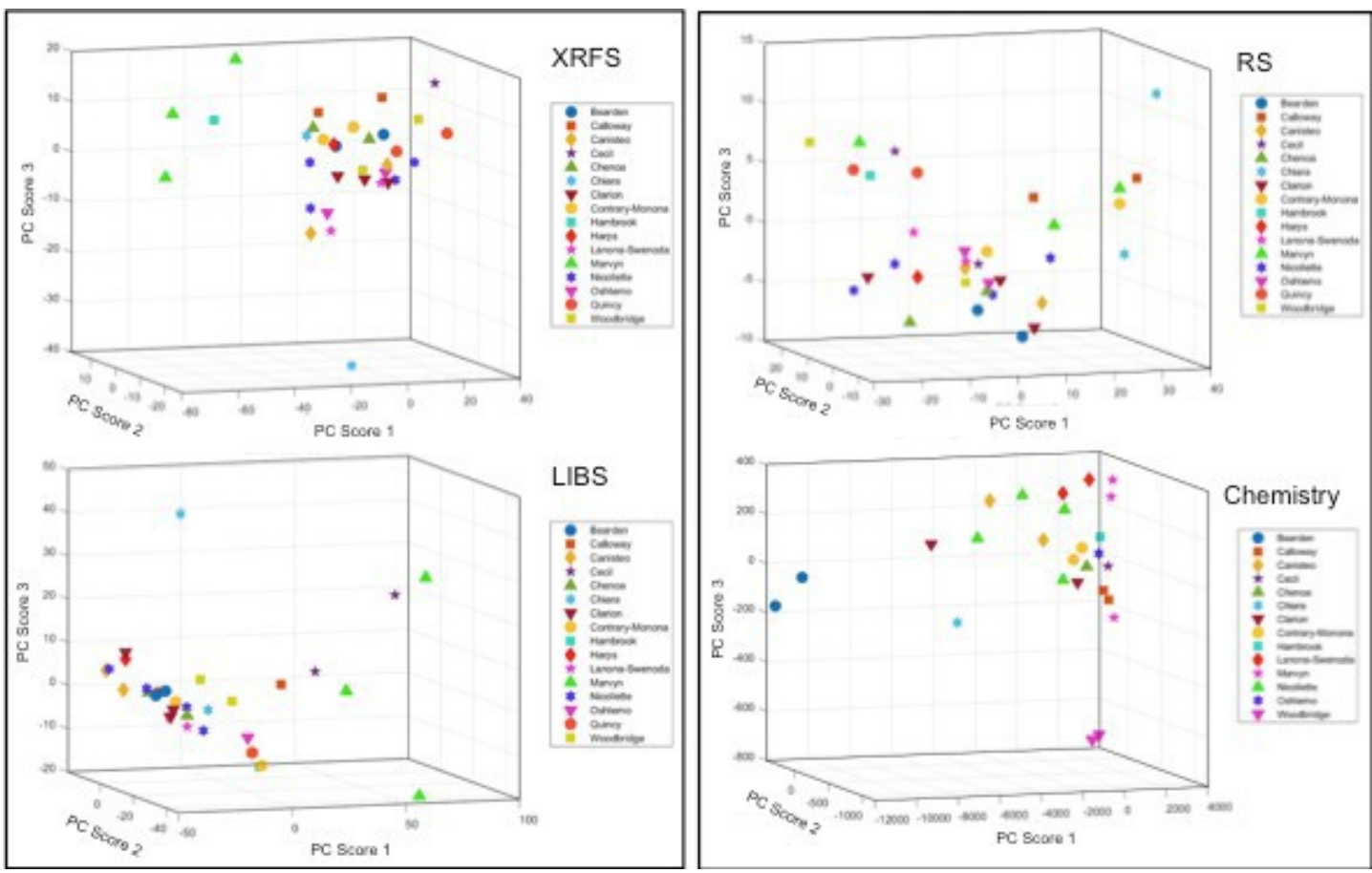

\subsection{New Hampshire soils}

Six samples were collected from the upper $5 \mathrm{~cm}$ of soil at two locations in central New Hampshire by JRPA, three samples from the USACE-ERDC Cold Regions Research \& Engineering Laboratory in Lebanon, $\mathrm{NH}$ and 
three samples from the Bean Hill locality in Belmont, NH. Additionally, three soil pits were excavated by SoilHydrology Associates at each of the Bean Hill sample sites, with the different soil horizons in each pit sampled and described.

Laboratory analysis was first undertaken for soil pellets prepared for the Lebanon/CRREL and Belmont soil sets and then for the soil pits at the Belmont location. The fusion feature vectors for the XRFS, RS, LIBS spectral data were reduced to 2-dimensions using PCA. This analysis demonstrated that the multi-analyzer approach can distinguish between different soil types and identify soils that are similar (Figure 20). Laboratory analysis of pellets from the three soil pits at Bean Hill in Belmont (Figure 21) documents the clear distinction of the ash and charcoal layers and other layers in soil pit and the compositional differences from the upper to lower soil layers.

Figure 20. Principal component analysis scores plot from laboratory analysis of the Lebanon/CRREL and Belmont soil sets.

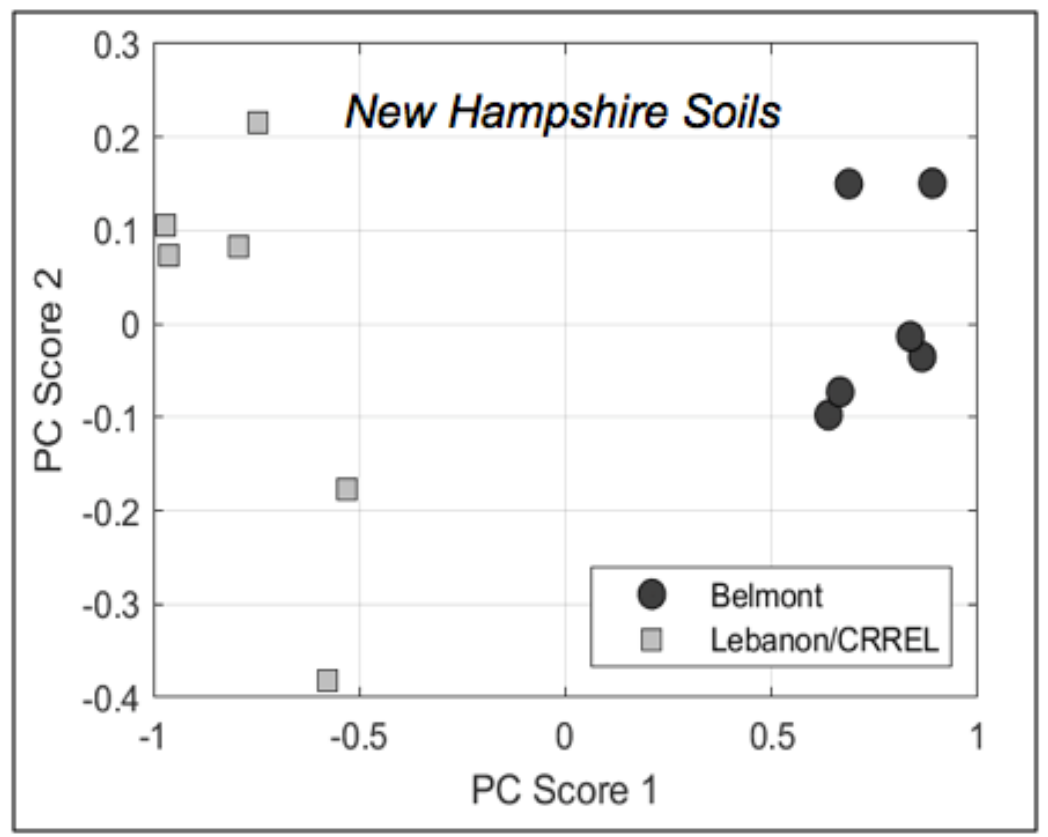


Figure 21. Principal components scores plot from laboratory analysis of the Belmont soil pits.

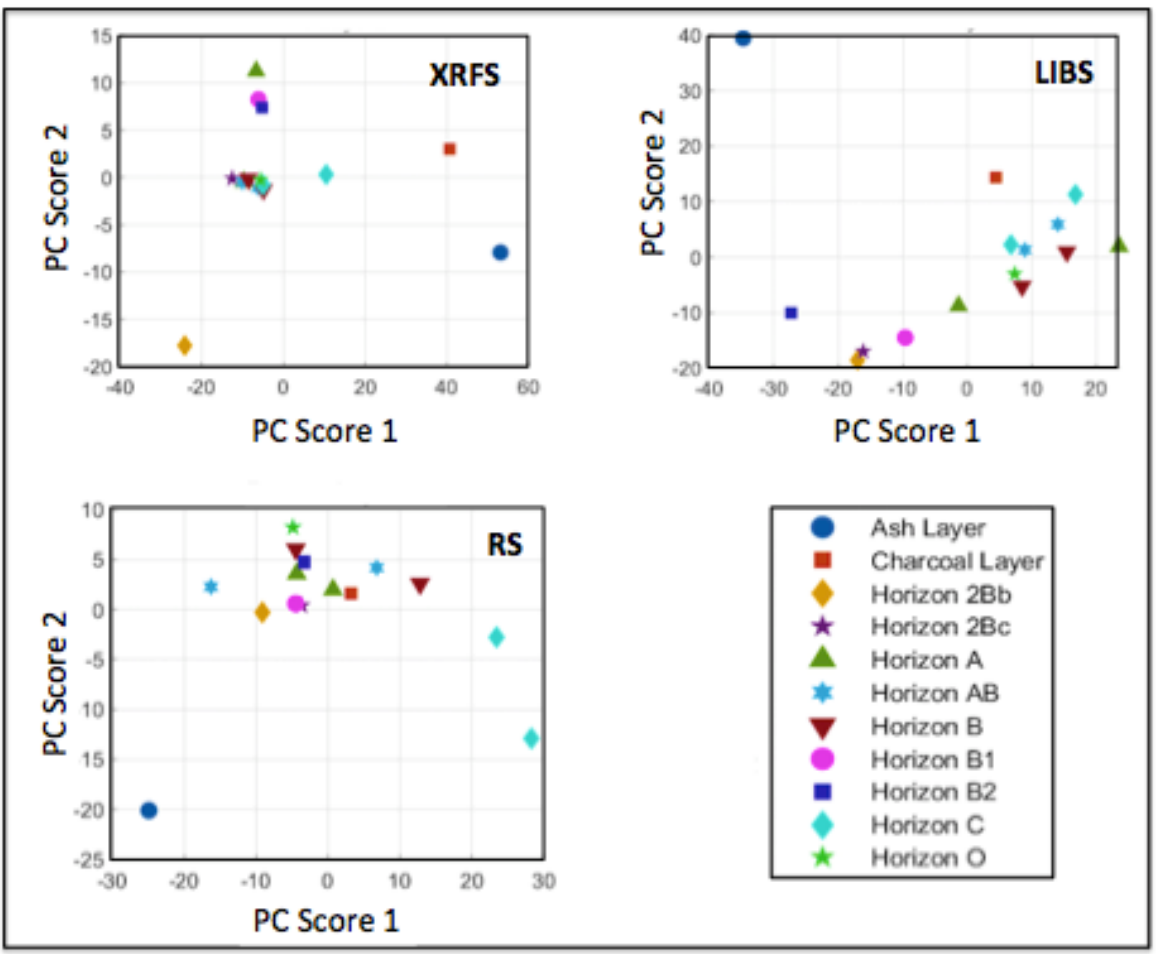

Classification for each individual analyzer and their spectral data fusion is shown on the right side of Figure 22. Discrimination between the Lebanon/CRREL and Belmont/Bean Hill soil sets is $100 \%$ correct for the XRFS, RS, and LIBS analyzers individually. Therefore, nothing can be gained through sensor fusion. By contrast, there is sufficient compositional variability for the different layers of the three Bean Hill soil pits, shown on the left side of Figure 22, that pit discrimination classification is extremely poor for the RS spectra $(<30 \%$ correct) and not strong for either LIBS $(<70 \%$ correct) or XRFS $(<80 \%)$ spectra. Similarly, in this second instance, data fusion does not provide any classification benefit.

Because the ultimate objective of this project was to develop a robust capability that could be used in the field, a short field study was conducted at the Belmont, New Hampshire site to test the performance of the handheld analyzers under field conditions with real time data transmission to a base station. XRFS and LIBS analyses were undertaken for this purpose; a logistical issue prevented use of RS analyzer. Field measurements in the three Bean Hill soil pits (Figure 23) demonstrated that the XRFS and LIBS perform with confidence under ambient environmental conditions, which included a rain shower during the fieldwork. 
Figure 22. Classification accuracy for the New Hampshire soil sets

from Belmont/Bean Hill (left) and Lebanon/CRELL (right)

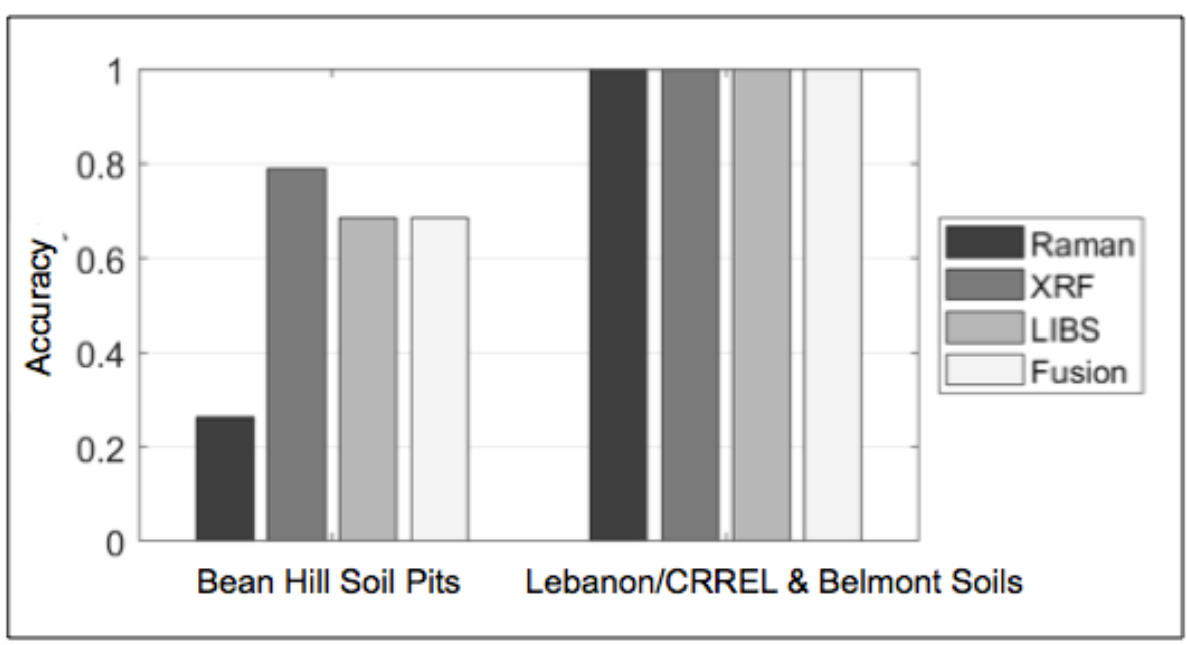

Figure 23. Principal component analysis scores plot for fused XRFS and LIBS spectral data sets for in-situ analysis of the three Belmont/Bean Hill soil pits.

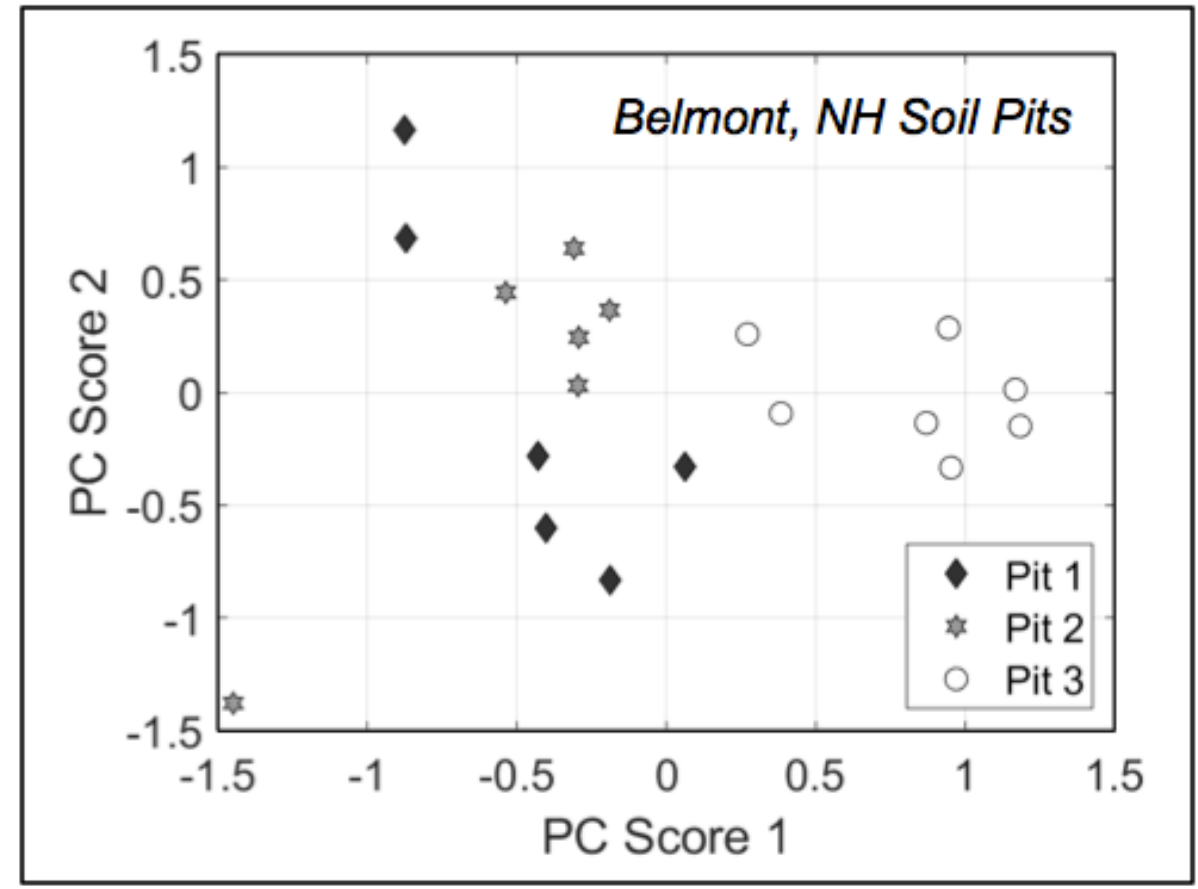




\section{Summary}

Funded under US Army ERDC-CRREL contract W913E518Coo11, an 18month multidisciplinary effort was undertaken by JRPlumer \& Associates, LLC between September 2018 to March 2020. This project had three technical objectives: (i) to upgrade current handheld technology for chemical analysis by X-ray fluorescence spectroscopy (XRFS), Raman spectroscopy (RS), and laser-induced breakdown spectroscopy (LIBS); (ii) to design a multisensor system based on these technologies for the rapid, in-situ chemical analysis of soils and other materials of military interest; and (iii) to investigate the classification/discrimination performance benefit that might be achieved through advanced signal pre-processing and data fusion with XRFS, RS, and LIBS analyses acquired for four suites of natural soils. This initiative was supported by sub-contracts to SciAps, Inc., Signal Analysis Solutions, LLC, SoilHydrology Associates, LLC, and Applied Spectra, Inc. Accomplishments of the program in the latter area are described in this report.

A total of 272 soils from four sources were analyzed for the project - 50 rangeland soil samples from central New Mexico, 21 soil samples from two sites in west-central New Hampshire, a suite of 144 agricultural soils from across the United States and Canada, and 58 soils from small arms ranges on military installations in Massachusetts, Virginia, Georgia, Idaho, Washington, and Alaska. An investigation of spectral data preprocessing was undertaken that examined the benefit that could be realized from Ar emission line removal from LIBS spectra, spectra baseline correction, spectral truncation and normalization, spectral culling through signal-to-noise ratio analysis, similarity analysis, uncertainty mitigation and adaptive quantization, and outlier rejection. A conceptual approach for real-time signal processing was developed and demonstrated through a field test. Finally, a hierarchical classification process was developed to combine spectral data from multiple analyzers and this was applied to the soil data acquired during the project. Using data from multiple collection dates it was observed that fusion of XRFS-RS-LIBS spectral data provided better classification/discrimination performance than for analysis by any individual analyzer. 


\section{References}

Das, B.S., Hendrickx, J.M.H., and Borchers, B. (2001). "Modeling transient water distributions around landmines in bare soils." Soil Science 166: 163-173.

Davies, L. and Gather, U. (1993). “The identification of multiple outliers.” Journal of the American Statistical Association 88: 782-792.

Egli, M., Hunt, A.G., Dahms, D., Raab, G., Derungs, C., Raimondi, S. and Yu, F. (2018). "Prediction of soil formation as a function of age using the percolation theory approach.” Frontiers in Environmental Science 6: 108.

Hempstalk, K., Frank, E., and Witten, I.H. (2008). "One-class classification by combining density and class probability estimation." In Proceedings of the Joint European Conference on Machine Learning and Knowledge Discovery in Databases. Antwerp, Belgium: Springer, 505-519.

Hendrickx, J.M.H., Alkov, N., Hong, S.-h., Van Dam, R.L., Kleissl, J., Shannon, H., Meason, J., Borchers, B., and Harmon, R.S. (2006). "New Mexico Tech landmine, UXO, IED detection sensor test facility: measurements in real field soils" In Proceedings of the International Society for Optical Engineering, SPIE, 6217: 278-289.

Jenny, H. (1994). Factors of Soil Formation: A System of Quantitative Pedology. New York: Dover Publications, $191 \mathrm{pp}$.

Kruse, F.A., Lefkoff, A.B., Boardman, J.W., Heidebrecht, K.B., Shapiro, A.T., Barloon, P.J., Goetz, A.F.H. (1993). "The spectral image processing system (SIPS)interactive visualization and analysis of imaging spectrometer data." Remote Sensing of the Environment 44: 145-163.

Merritts, D.J., Chadwick, O.A., and Hendricks, D.M. (1991). "Rates and processes of soil evolution on uplifted marine terraces, Northern California." Geoderma 51: 241275 .

Miller, T.W., Hendrickx, J.M.H., and Borchers, B. (2004). "Radar detection of buried landmines in field soils." Vadose Zone Journal 3: 1116-1127.

NRCS (1999). Soil Taxonomy - A Basic System of Soil Classification for Making and Interpreting Soil Surveys. Washington, DC: US Department of Agriculture, Natural Resources Conservation Service.

Otsu, N. (1979). "A threshold selection method from gray-level histogram.” IEEE Transactions on Systems, Man, and Cybernetics 8: 62-66.

Soil Survey Staff (2014). Keys to Soil Taxonomy, 12th Edition. United States Department of Agriculture, Natural Resources Conservation Service, 372 pp.

Van Dam, R.L., Hendrickx, J.M.H., Borchers, B., Hong, S.-h., Miller, T.W., and Harmon, R.S. (2004). "A controlled outdoor test site for evaluation of soil effects on land mine detection sensors.” In Proceedings of the International Society for Optical Engineering, SPIE, 5415: 1335-1344. 
White, A. G., Blum, A. E., Schulz, M. C., Bullen, T. D., Harden, J. W., and Peterson, M. L. (1996). "Chemical weathering rates of a soil chronosequence on granitic alluvium: I. quantification of mineralogical and surface area changes and calculation of primary silicate reaction rates." Geochimica Cosmochimica Acta, 60: $2533-2550$. 


\section{Appendix: The Soil Suites}

\section{Background}

A total of 272 soils from four sources were analyzed during the project analytical work in 2019. These are follows: (i) 50 rangeland soil samples from central New Mexico, (ii) 21 soil samples from two sites in west-central New Hampshire, (iii) a suite of 144 agricultural soils from across the United States and Canada provided by Colorado State University, and (iv) 58 soils from small arms ranges on military installations in Massachusetts, Virginia, Georgia, Idaho, Washington, and Alaska provided by USACECRREL. The paragraphs that follow describe each soil suite.

\section{The New Mexico Soil Suite}

Soils forming in arid environments (i.e. in areas receiving $<700 \mathrm{~mm}$ of precipitation annually) develop in a distinctly different way from those in wetter environments. Arid soils are characterized by the accumulation of eolian dust consisting of fine particles (silt and clay) and soluble salts (such as calcium carbonate and calcium sulfate plus sodium chloride in some hyperarid areas). Dust fluxes and composition vary greatly spatially, with some dust in the southwest United States arriving from as far away as China, while deposition tends to be high downwind of large playa lakes. The samples collected for this project reflect the input of eolian dust and also some of the issues pertaining to the relationships between soil taxonomic units and field mapping units that affect NRCS rangeland soil maps at spatial scales of 1:50,000 or greater.

Within the budgetary limits available for soil collection in New Mexico, it was not possible to select a truly representative small set of soils from such a large state of heterogeneous geological character. Therefore, instead of using a statistical approach for the sampling design, criteria were developed to test the capability of the multi-analyzer approach to distinguish different soil types and to identify similar soil types. These criteria, posed as questions, are: (i) Can the multi-analyzer approach detect which soils are different from one another?; (ii) Can the multi-analyzer approach detect which soils are the same?; and (iii) Can the multi-analyzer approach discriminate between soils that are somewhat different, but have common underlying characteristics? 
Initially, eight survey samples were collected from four soil series in central New Mexico by SoilHydrology Associates in August of 2018 for the development of a sampling protocol as well as initial testing and analysis by JRPA. The sampling protocol developed consisted of taking a sample of about $500 \mathrm{~g}$ from the top $0-5 \mathrm{~cm}$ of the soil (Fig. 6.1). After air-drying in the laboratory at room temperature, the samples were sieved to remove all particles and root debris exceeding a size of $2 \mathrm{~mm}$. The sieved samples were sent to JRPA for further processing and analysis.

This protocol was then followed to sample soils from six locations across central-north New Mexico in November and December 2018 Six samples were collected from each location along a straight transect approximately $22 \mathrm{~m}$ apart. Described in Table A1, six soils sampled for the project were: (i) the loamy fine sand of the Bluepoint Soil Series at the Rio Salado, (ii) the very stony sandy loam of the Laborcita-Pilabo-Lemitar Soil Series Complex in Upper Socorro Canyon, (iii) the very stony sandy loam of the Laborcita-Pilabo-Lemitar Soil Series complex in Lower Socorro Canyon, (iv) the gravelly sandy loam of the Millett-Sedillo Soil Series complex at Sedillo Hill, (v) the gypsiferous sand of the Lark-Transformer Soil Series association at White Sands, and (vi) the strongly saline silty clay loam of the playa soil at Willard. At Rio Salado, two sets of samples were gathered: one at depth $0-3 \mathrm{~cm}$ and one at $10-13 \mathrm{~cm}$. Thus, a total of 42 soil surface samples formed the primary suite of samples analyzed for the project.

Figure A1. Geographic locations of the six New Mexico soil sampling sites.

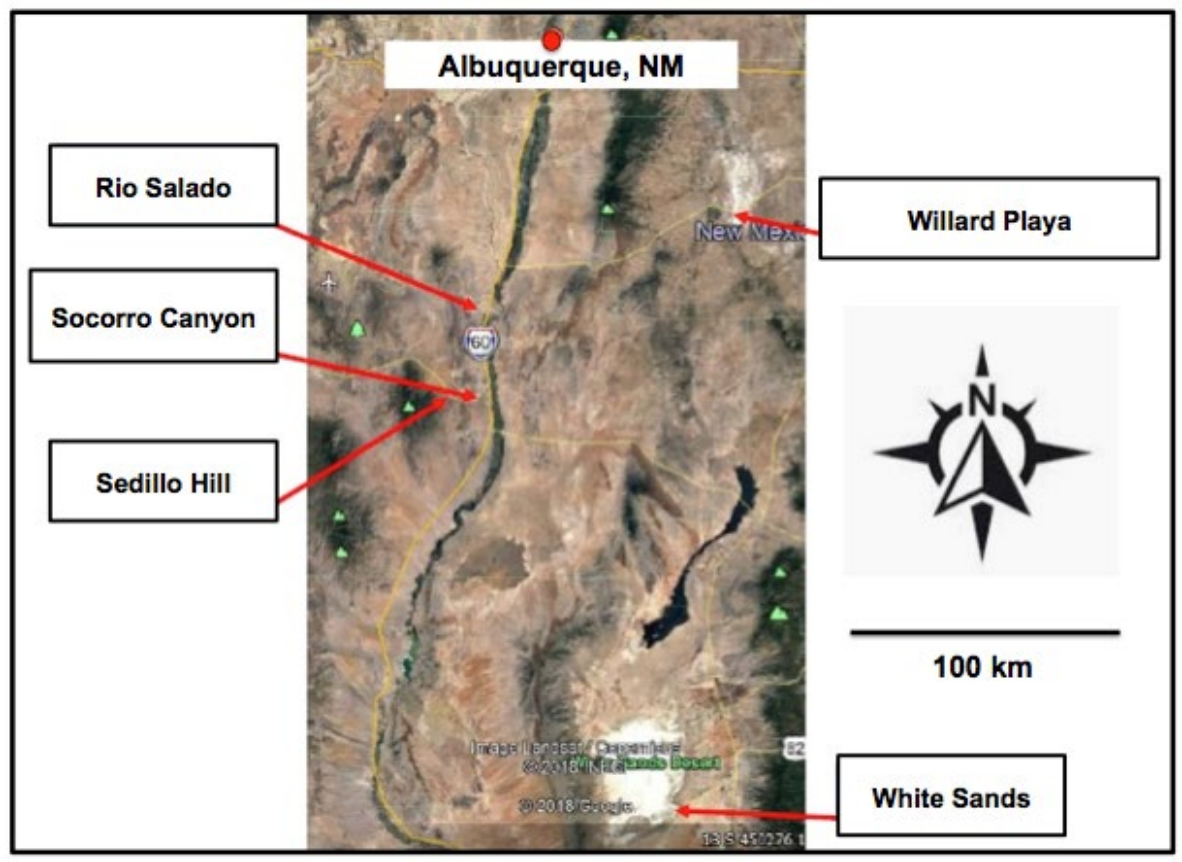


Soil series are classified not only based on the characteristics of the surface soil layer but on all soil horizons and characteristics down to a depth of 2 $m$ depth (Soil Survey Staff, 2014) Often, there is no direct relationship between the properties of the $0-3 \mathrm{~cm}$ soil surface layer and its soil series name. Nevertheless, in order to provide more understanding of the context of the soil samples used in this project a short description with visuals is presented below for each of the New Mexico soil locations.

Table A1. New Mexico soil sample sites.

\begin{tabular}{|c|c|c|c|}
\hline Location & Soil Series Name & Depth & Texture \\
\hline Sedillo Hill & 445-Millett-Sedillo complex & $0-10 \mathrm{~cm}$ & gravelly sandy loam \\
\hline Sedillo Hill & 445-Millett-Sedillo complex & $15-30 \mathrm{~cm}$ & gravelly loam \\
\hline Nogal Canyon & $\begin{array}{l}649-\text { Nickel-Caliza very gravelly sandy } \\
\text { loams }\end{array}$ & $0-5 \mathrm{~cm}$ & $\begin{array}{l}\text { very gravelly sandy } \\
\text { loam }\end{array}$ \\
\hline Nogal Canyon & $\begin{array}{l}\text { 649-Nickel-Caliza very gravelly sandy } \\
\text { loams }\end{array}$ & $0-5 \mathrm{~cm}$ & $\begin{array}{l}\text { very gravelly sandy } \\
\text { loam }\end{array}$ \\
\hline Rio Salado & 620-Bluepoint loamy fine sand & $0-5 \mathrm{~cm}$ & loamy fine sand \\
\hline Rio Salado & 620-Bluepoint loamy fine sand & $0-5 \mathrm{~cm}$ & loamy fine sand \\
\hline Socorro Canyon & 621-Arizo-Riverwash complex & $5-10 \mathrm{~cm}$ & $\begin{array}{l}\text { very gravelly coarse } \\
\text { sand }\end{array}$ \\
\hline Socorro Canyon & 621-Arizo-Riverwash complex & $0-5 \mathrm{~cm}$ & gravelly sandy loam \\
\hline
\end{tabular}

Seven sets of six samples were collected to determine if the multi-analyzer approach could distinguish different soils and identify similar soils. The two sets at Rio Salado contain the same soil to test whether these samples would be identified as similar. All other soils are different one from the other and from the Rio Salado soil to test whether these would be identified as different soils. Three soils differ only by age: Sedillo Hill (740,000 years), Socorro Canyon Upper (140,000 years), and Socorro Canyon Lower (25,000 years). These soils were sampled to test whether soils that are different in some aspects (i.e. age) but are similar in other aspects (i.e. parent material, climate, flora and fauna, and topography) could be discriminated.

\section{Bluepoint Soil Series}

NRCS description: The Bluepoint Soil Series consists of very deep, somewhat excessively drained soils that formed on dunes and sand sheets in eolian materials derived from mixed rock sources. The typical NRCS profile for the Bluepoint Series is: 
$\mathrm{C} 1-\mathrm{o}$ to 5 inches: loamy fine sand,

C2 -5 to 28 inches: loamy fine sand,

$\mathrm{C}_{3}-28$ to 53 inches: loamy fine sand, and

$\mathrm{C}_{4}-53$ to 60 inches: loamy sand.

The soil at the project sampling location at the Rio Salado (Figs. 5.2.4 and 5.2.5) is indeed formed in a sand sheet on the south bank of the Rio Salado River north of Socorro. The soil map unit is located on the top of a large fan with different soil series present on the incised slopes. The boundary on the NRCS soil map is reasonably located for this soil series. The NRCS description indicates that the soil contains gypsum, but no gypsum was present in this profile excavated; instead incipient calcium carbonate accumulation was observed. A buried soil was identified within this soil, with the top $40 \mathrm{~cm}$ of the profile being very recent sand overlying a more strongly developed soil also forming within the sand.

A soil chronosequence describes portions of a soil series that have developed in a similar parent material, under a similar climate, with similar flora and fauna, and on a similar landform but differ in their degree of profile development only because of differences in age. Numerous studies have shown systematic changes in most soil properties (physical and chemical) with increasing soil age (e.g. White et al., 1996; Merrits et al., 1991; Egli et al., 2018). The soil series sampled near Socorro, NM form a chronosequence. The youngest soil at Lower Socorro Canyon (Arizo Series and the Arizo-Riverwash complex) is approximately 25,000 years old, the soil at Upper Socorro Canyon (Nolam Series) is about 140,000 years old, and the soil at Sedillo Hill (Ladron Series and the Millett-Sedillo complex) is approximately 750,000 years old. The NRCS soil map locates a Rock Outcrop soil series 786, which is described as bedrock, just north of the project sampling site. Yet, there is no bedrock at this location, instead the parent material for the soil are fluvial gravels from the Rio Salado that are overlain by young sand sheets that have been mobile within historic time. Such discrepancies between the general NRCS description of a soil series and the actual characteristics of a soil at any particular place are not uncommon. The explanation lies in the basic heterogeneous character of natural soils. Thus there can be a distinct difference between the representative profile for a soil series description selected at one specific place and the character of the same soil at any other place, often tens or hundreds of kilometers distant. The two different Socorro Canyon soil series are separated by a well-defined boundary at the Socorro Canyon Fault, across which there is a $2 \mathrm{~m}$ difference in elevation. The Arizo-Riverwash 
Complex soil series is present on the Lower Socorro Canyon surface, whereas the Upper Socorro Canyon surface is covered by the Nolam soil series.

\section{Arizo-Riverwash Complex at Socorro}

NRCS description: The Arizo-Riverwash Complex at Socorro Canyon (Figures 6.6-6.9) consists of the Arizo Soil Series and similar soils (55\%), gravelly Riverwash (30\%), and minor other soil components (15\%). The typical NRCS profile is:

A - o to 2 inches: gravelly sandy loam,

$\mathrm{C}-2$ to 60 inches: very gravelly coarse sand.

NRCS description: The Arizo Soil Series consists of very deep, excessively drained soils that formed in mixed alluvium. These soils are found on recent alluvial fans, inset fans, fan apron, fan skirts, stream terraces, floodplains of intermittent streams and channels. The Arizo series is classified as an Entisol, one of the 12 soil orders, meaning that it very weakly weathered, which indicates a relatively young age. The typical soil profile used by the NRCS to describe this soil series is located in Nevada. The typical NRCS profile for the Arizo Soil Series is:

A - o to 3 inches: very stony loamy sand,

$\mathrm{C}-3$ to 60 inches: stratified cobble-rich coarse sand to extremely gravelly loamy sand.

Our description of the Arizo soil on the Lower Socorro Canyon surface has quite a different horizonation. The subscripts indicate the secondary characteristics of the master horizon. In the nomenclature used for classification, $v$ is vesicular (meaning it has numerous vesicles or holes in the ped), $w$ is weak (indicating a subtle change in color), and $k$ is calcium carbonate accumulation (indicated by color or reaction to hydrochloric acid). The local profile of the Arizo soil where sampled is:

Av - o to $6 \mathrm{~cm}$ : Dark brown (7.5YR 3/3) gravelly loamy sand, Bw1 -6 to $22 \mathrm{~cm}$ : Brown (7.5YR 4/4) gravelly loamy sand, Bw2 - 22 to $8 \mathrm{~cm}$ : Dark yellowish brown (10YR 4/4) gravelly sandy loam,

Bw3 - 22 to $38 \mathrm{~cm}$ : Brown (7.5YR 4/4) gravelly loamy sand, Bw4 - 57 to $85 \mathrm{~cm}$ : Olive brown (2.5Y 4/4) Gravelly sand, $\mathrm{Ck}-85$ to $94 \mathrm{~cm}$ : Light olive Brown (2.5Y5/6) Gravelly sand. 


\section{Nolam Soil Series at Socorro Canyon}

NRCS description: The Nolam Soil Series (Figs. 6.6 to 6.8) consists of very deep, well drained, moderately slow permeable soils that formed in alluvial sediments derived from rhyolite and andesite on terraces and piedmonts. The typical NRCS profile is:

A - o to 2 inches: gravelly sandy loam,

Bt - 2 to 13 inches: very gravelly sandy clay loam,

$\mathrm{Ck}-13$ to 60 inches: very gravelly sandy loam.

The description of the Nolam Soil Series on the NRCS map is also different than what was observed in Socorro Canyon Upper surface at this site, where the local soil profile was mapped as follows:

Av - o to $5 \mathrm{~cm}$ : Dark Brown (7.5YR 3/4) Gravelly Sandy Loam, $\mathrm{AB}-5$ to $31 \mathrm{~cm}$ : Dark Brown (7.5YR 3/4) Gravelly Sandy Loam, $\mathrm{Bt}-31$ to $58 \mathrm{~cm}$ : Yellowish red (5YR 4/6) Very Gravelly sandy clay loam,

Btk - 58 to $75 \mathrm{~cm}$ : Red (2.5YR 4/6) Very gravelly sandy clay loam, $\mathrm{K}-75$ to $152 \mathrm{~cm}$ : Reddish yellow (5YR6/8) very gravelly sandy loam, Ck - 152 to $180 \mathrm{~cm}$ : Light olive brown (2.5YR 5/4 Very Gravelly loamy sand.

The K horizon designation is not used by the NRCS; however, it is widely used by soil geomorphologists to indicate a soil horizon in which all the properties are determined by the degree of calcium carbonate cementation. The horizon is indurated with a particularly slow unsaturated hydraulic conductivity.

In addition to the differences between the NRCS typical profiles and the local profiles observed in Socorro Canyon, there is one important additional local observation that pertains to the location of the boundary between the Arizo and Nolam series shown on the NRCS soil map (Fig. 6.6). The orange boundary presented on the map is not correct, as the actual boundary is located along the fault line (i.e. the black line on the map). This is the nature of a 1:50,000 scale soil map and the issue of boundary location on such maps. This problem of misplaced soil boundary locations on such large-scale maps is not well understood by the portion of the soil science community who lack geomorphological expertise. 


\section{Ladron Soil Series at Sedillo Hill}

NRCS description: The Ladron Soil Series (Figs. 6.10 and 6.11) consists of deep, well-drained, moderately permeable soils that formed in alluvium. These soils form on knolls and fan terraces. The typical soil profile is:

A - o to 2 inches: very gravelly sandy loam

Bw -2 to 31 inches: very gravelly loam

Bk1 - 31 to 47 inches: very gravelly sandy loam

Bk2 - 47 to 60 inches: very gravelly loam

\section{Millett-Sedillo Complex at Sedillo Hill}

NRCS description: The Millett-Sedillo Complex at Sedillo Hill (Figures 5.10 and 5.11) consists of the Millett and similar soils (50 \%), the Sedillo and similar soils (30 \%), and the Minor other soil components (20\%).

NRCS description: The Millett Soil Series consists of very deep, welldrained soils formed in alluvium weathered from old gravelly alluvium known as 'rim gravels'. This material is composed of quartzite, sandstone and some basic igneous rocks. Millett soils are on stream and fan terraces on plateaus and footslopes and shoulders on stable landslides. The typical soil profile is:

A - o to 3 inches: gravelly sandy loam, Bt - 3 to 18 inches: gravelly loam, Bk - 18 to 60 inches: very gravelly sandy loam.

NRCS description: The Sedillo Soil Series consists of very deep, welldrained, moderately slowly permeable soils that formed in gravelly alluvium. Sedillo soils are on bajadas, piedmonts, fan terraces, fan remnants, and stream terraces. The typical profile is:

A - o to 3 inches: very gravelly fine sandy loam, $\mathrm{Bt}-3$ to 19 inches: very gravelly sandy clay loam, Bk - 19 to 60 inches: very gravelly fine sandy loam.

The three NRCS typical profiles presented above also differ considerably from observations made during the project fieldwork. The local soil profile is:

Ah - o to $7 \mathrm{~cm}$ : Brown (7.5YR 4/4) Sandy Loam, 
$\mathrm{AB}-7$ to $27 \mathrm{~cm}$ : Dark Brown (7.5 YR 3/3) Gravelly Loam, Bt1 - 27 to $42 \mathrm{~cm}$ : Dark Reddish Brown (5YR 3/4) Gravelly Sandy Clay Loam, Btk - 42 to $55 \mathrm{~cm}$ : Red (2.5YR 4/6) Gravelly Silty Clay Loam, K1 -55 to $69 \mathrm{~cm}$ : Reddish Yellow (5YR 6/9) Gravelly Sandy Clay Loam, K2 - 69 to $112 \mathrm{~cm}$ : Light Red (2.5YR 6/6) Gravelly Sandy Clay Loam.

\section{Lark-Transformer Association Soil Series at White Sands}

NRCS description: The Lark-Transformer Association Soil Series (Figs 6.12 and 6.13) consists of very deep, excessively-drained soils that formed in sandy gypsiferous eolian deposits. The typical soil profile is:

Cyy1 - o to 2 inches: gypsiferous sand,

Cyy2 - 2 to 41 inches: gypsiferous sand,

Cyy3 - 41 to 60 inches: gypsiferous sand.

No other field observations were made at this site.

\section{The Playa Soil at Willard}

The final soil sampled for the project was the strongly saline silty clay loam of the playa soil at Willard, NM (Figs 6.14 and 6.15). The typical soil profile here is:

H1 - o to 6 inches: silty clay loam, H2 - 6 to 60 inches: clay.

No other field observations were made at this site.

\section{The New Hampshire Soil Suite}

Six samples were collected from the upper $5 \mathrm{~cm}$ of soil at two locations in central New Hampshire by JRPA, three samples from the USACE-ERDC Cold Regions Research \& Engineering Laboratory in Lebanon, NH and three samples from the Bean Hill locality in Belmont, NH. Additionally, three soil pits were excavated by SoilHydrology Associates at each of the Bean Hill sample sites, with the different soil horizons in each pit sampled and described. The three soils described and analyzed at the Bean Hill during the week of 6-12 August 2019 have been mapped as belonging to three NRCS soil series - Canterbury Fine Sandy Loam (167B), Moosilauke fine sandy loam (415 B), and Pillsbury Fine sandy loam (647B). Due to the 
scale of the NRCS maps the actual soils sampled described at the Bean Hill site may not necessarily fall within these NRCS soil series.

\section{Soil at Bean Hill Site 1}

The soil at site BH-1 (Fig. 6.17) is mapped as the Pillsbury FSL (647B). This soil is located on a small boulder terrace adjacent to a small stream. Soil horizons at the site are as follows:

- Litter 10-0 cm,

- Ah horizon @ 0-10 cm,

- $\mathrm{AB}$ horizon@ 10-16 cm,

- Bw horizon @ 16-24 cm,

- C horizon@24-55 cm.

\section{Soil at Bean Hill Site 2}

The soil at site BH-2 is mapped as the Canterbury FSL (167 B). This soil is located in the upper backslope position on a heavily forested drumlin. Soil horizons at the site are as follows:

- Litter@6-o cm,

- $\quad$ Ah horizon@ 0-18 cm

- $\mathrm{AB}$ horizon @ 18-25 cm,

- Bw horizon @ 25-34 cm,

- C horizon @ 35-65 cm.

\section{Soil at Bean Hill Site 3}

The Soil at site BH-3 (Figure 6.19) is mapped as Moosilauke FSL (415B). This soil is located at the base of a small drumlin on a lower footslope position. Soil horizons at the site are as follows:

- Litter @ 5-o cm,

- $\quad$ Ah@ 0-10 cm,

- Bwj1@10-25 cm,

- Bwj2@ 25-36 cm,

- $\quad$ Ash and charcoal layer varying between o-8 $\mathrm{cm}$ thick,

- $\quad 2 B w b @ 7 \mathrm{~cm}$,

- $\quad 2 \mathrm{Cb} @ 12 \mathrm{~cm}$.

The first two soils at the $\mathrm{BH}-1$ and $\mathrm{BH}-2$ sites appear similar in terms of degree of development and, therefore, probably in age. They are forming 
in well-sorted fine sand that contains occasional large boulders. Weathering of these soils has produced some variation in soil properties with depth, but the soils are overall weakly developed and probably of Late Pleistocene age. The third soil at the $\mathrm{BH}-3$ site displays a compound soil profile, in that it contains a buried soil. The soil in the upper stratigraphic layer is less strongly weathered than at the $\mathrm{BH}-1$ and $\mathrm{BH}-2$ sites. The soil beneath the ash layer is probably equivalent in age to the soil at the $\mathrm{BH}-1$ and $\mathrm{BH}-2$ sites. The soil pit at the $\mathrm{BH}-3$ site contained a compound buried soil, the upper soil is weakly developed and it overlies the buried soil which has evidence of a previous fire and a buried soil beneath the ash and charcoal. Burial appears to be localized, as the depth of the most recent stratigraphic layer is unlikely to have come upslope. A large depression located adjacent to this soil pit is suggested as the source of the burial soil.

\section{The CSU Soil Suite}

Through an arrangement with Dr. Robert Miller of the Department of Crop and Soil Science at Colorado State University (CSU) a collection of 166 agricultural soils were provided to the project that were selected from the ALP Laboratory Proficiency Program (ALP-LPP) archive, of which 144 were analyzed during the course of this project. The soils of the ALPLPP program were collected across 32 US states and 7 Canadian provinces. The samples received, which had been milled to $<0.7 \mathrm{~mm}$ and homogenized, were from the top $20 \mathrm{~cm}$ of the soil profile at the selected US and Canadian sampling locations. The US soils, which are from all 12 of the NRCS soil orders, are particularly well characterized compositionally through analytical work undertaken by the ALP-LPP program. Analysis of the first 96 samples of this suite was undertaken in January 2019, with a subsequent 48 samples analyzed in August 2019. These 144 soils represent 10 of the 12 NRCS Soil Orders, but three of the classes are only represented by a single sample and another only has two samples. For purposes of the statistical analysis undertaken by subcontractor Signal Analysis Solutions (see Sections 7 and 8 below), analytical data for the CSU sample suite was grouped for processing according to five different schema: Soil Order, Thermal Regime, Moisture Regime, Mineralogical Character, and Geological Source.

\section{The Military Installation Soil Suite}

An additional 58 soil samples came from six US military installations (Ft. Wainwright, AK; Ft Lewis, WA, Idaho National Guard Camp Kinama; Ft. 
Benning, GA; Ft. Eustis, VA; and Massachusetts Military Reservation/Joint Base Cape Cod). Provided by Dr. J. Clausen of the USACEERDC Cold Regions Research and Engineering Laboratory (CRREL), these soils were collected from the top $2.5 \mathrm{~cm}$ of soil at small arms firing ranges and, therefore, are contaminated to various extents with metal elements present in munitions (such as $\mathrm{Cu}, \mathrm{Zn}, \mathrm{Pb}, \mathrm{W}$, and $\mathrm{Sb}$ ). A range of soil compositions, varying from uncontaminated to strongly contaminated, were analyzed for each of the six military installations. The NRCS Soil Series to which these soils are assigned is not known. These six military installation soil suites were processed during the March-April 2019 period of analytical work. 


\section{Acronyms}

\begin{tabular}{|c|c|}
\hline ASI & Applied Spectra, Inc. \\
\hline COTS & Commercial-off-the-shelf \\
\hline $\mathrm{CRM}$ & Certified reference material \\
\hline CRREL & Cold Regions Research and Engineering Laboratory \\
\hline $\mathrm{CSU}$ & Colorado State University \\
\hline ERDC & Engineer Research and Development Center \\
\hline $\mathrm{eV}$ & Electron volt \\
\hline FWHM & Full width at half maximum \\
\hline GUI & Graphical user interface \\
\hline $\mathrm{Hz}$ & Hertz \\
\hline ICP-MS & Inductively coupled plasma mass spectrometry \\
\hline JRPA & JRPlumer \& Associates, LLC \\
\hline $\mathrm{KeV}$ & Kilo electron volt \\
\hline LA-ICP-MS & $\begin{array}{l}\text { Laser ablation inductively coupled plasma mass spec- } \\
\text { trometry }\end{array}$ \\
\hline LIBS & Laser-induced breakdown spectroscopy \\
\hline LOSO & Leave-one-sample-out \\
\hline$\mu$ & Micron \\
\hline$\mu \mathrm{m}$ & Micrometer \\
\hline $\mathrm{mA}$ & Milliamp \\
\hline $\mathrm{mJ}$ & millijoule \\
\hline $\mathrm{mm}$ & Millimeter \\
\hline $\mathrm{ns}$ & Nanosecond \\
\hline $\mathrm{PC}$ & Principal component \\
\hline PCA & Principal component analysis \\
\hline $\mathrm{QA} / \mathrm{QC}$ & Quality assurance/Quality control \\
\hline NIST & National Institute of Science and Technology \\
\hline $\mathrm{nm}$ & Nanometer \\
\hline NOTA & None of the above \\
\hline NRCS & Natural Resources Conservation Service \\
\hline $\mathrm{R} \& \mathrm{D}$ & Research and development \\
\hline RDTE & Research, Development, Test and Evaluation \\
\hline $\mathrm{RS}$ & Raman spectroscopy \\
\hline RSD & Relative standard deviation \\
\hline SAS & Signal Analysis Solutions, LLC \\
\hline SciAps & SciAps Inc. \\
\hline SHA & SoilHydrology Associates, LLC \\
\hline SMU & Soil mapping unit \\
\hline SNR & signal-to-noise ratio \\
\hline USACE & U.S. Army Corps of Engineers \\
\hline USDA & U.S. Department of Agriculture \\
\hline XRFS & X-ray fluorescence spectroscopy \\
\hline
\end{tabular}




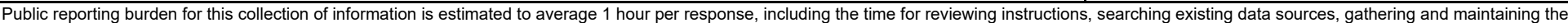

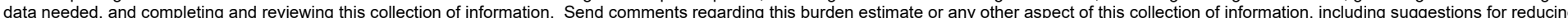

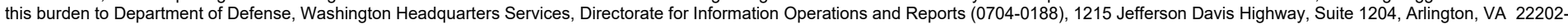

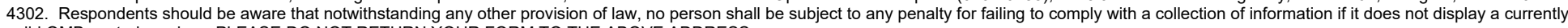
valid OMB control number. PLEASE DO NOT RETURN YOUR FORM TO THE ABOVE ADDRESS.

\begin{tabular}{l|l|l} 
1. REPORT DATE & 2. REPORT TYPE & 3. DATES COVERED (From - To)
\end{tabular} Final

\section{TITLE AND SUBTITLE}

Fusion of Spectral Data from Multiple Handheld Analyzers (LIBS, XRF and Raman) for Chemical Analysis and Classification of Soil

\section{5a. CONTRACT NUMBER}

W913E518C0011

5b. GRANT NUMBER

5c. PROGRAM ELEMENT NUMBER

5d. PROJECT NUMBER

474428

5e. TASK NUMBER

5f. WORK UNIT NUMBER

8. PERFORMING ORGANIZATION REPORT NUMBER

ERDC/ CRREL CR-20-1

JRPlumer Associates, LLC

Gilford, NH 03249

\section{SPONSORING / MONITORING AGENCY NAME(S) AND ADDRESS(ES)}

10. SPONSOR/MONITOR'S ACRONYM(S)

U.S. Army Engineer Research and Development Center (ERDC)

Cold Regions Research and Engineering Laboratory

52 Lyme Road

Hanover, NH 03755
11. SPONSOR/MONITOR'S REPORT NUMBER(S)

\section{DISTRIBUTION / AVAILABILITY STATEMENT}

Approved for public release; distribution is unlimited.

\section{SUPPLEMENTARY NOTES}

\section{ABSTRACT}

An 18-month multidisciplinary project was undertaken by JRPlumer \& Associates, LLC and four subcontractors that had three technical objectives: (i) to upgrade current handheld technology for chemical analysis by X-ray fluorescence spectroscopy (XRFS), Raman spectroscopy (RS), and laser-induced breakdown spectroscopy (LIBS); (ii) to design a multisensor system based on these technologies for the rapid, in-situ chemical analysis of soils and other materials of military interest; and (iii) to investigate the classification/discrimination performance benefit that might be achieved through advanced signal pre-processing and data fusion with XRFS, RS, and LIBS analyses acquired for four suites of natural soils. Accomplishments of the program in the latter area are described in this report.

\section{SUBJECT TERMS}

Scientific apparatus and instruments, Chemistry, Analytic, Soils-Testing, X-ray spectroscopy, Raman spectroscopy, Laser-induced breakdown spectroscopy

\section{SECURITY CLASSIFICATION OF:}

\section{a. REPORT}

Unclassified

\section{b. ABSTRACT} Unclassified c. THIS PAGE Unclassified

\begin{tabular}{c|c|c|}
$\begin{array}{c}\text { 17. LIMITATION } \\
\text { OF ABSTRACT }\end{array}$ & $\begin{array}{c}\text { 18. NUMBER } \\
\text { OF PAGES }\end{array}$ & \\
\cline { 2 - 3 } UU & 65 & \\
& &
\end{tabular}

19a. NAME OF RESPONSIBLE PERSON

19b. TELEPHONE NUMBER 